\title{
Defining the Value of Injection Current and Effective Electrical Contact Area for EGaln-Based Molecular Tunneling Junctions
}

\section{Citation}

Simeone, Felice C., Hyo Jae Yoon, Martin M. Thuo, Jabulani R. Barber, Barbara Smith, and George M. Whitesides. 2013. "Defining the Value of Injection Current and Effective Electrical Contact Area for EGaln-Based Molecular Tunneling Junctions." Journal of the American Chemical Society 135(48): 18131-18144.

\section{Published Version}

doi:10.1021/ja408652h

\section{Permanent link}

http://nrs.harvard.edu/urn-3:HUL.InstRepos:12361259

\section{Terms of Use}

This article was downloaded from Harvard University's DASH repository, and is made available under the terms and conditions applicable to Open Access Policy Articles, as set forth at http:// nrs.harvard.edu/urn-3:HUL.InstRepos:dash.current.terms-of-use\#OAP

\section{Share Your Story}

The Harvard community has made this article openly available.

Please share how this access benefits you. Submit a story.

Accessibility 


\title{
Defining the Value of Injection Current and Effective Electrical Contact Area
}

\section{for EGaIn-based Molecular Tunneling Junctions}

\author{
Felice C. Simeone, Hyo Jae Yoon, Martin M. Thuo, Jabulani R. Barber, Barbara Smith, \\ and George M. Whitesides*
}

Department of Chemistry and Chemical Biology, Harvard University, 12 Oxford Street, Cambridge, Massachusetts 02138 United States

\footnotetext{
*Corresponding author

Tel.: +1 6174589430

Fax.: + 16174589857

e-mail: gwhitesides@gmwgroup.harvard.edu
} 


\section{Abstract}

For SAMs of $n$-alkanethiolates $\mathrm{SC}_{\mathrm{n}}$ (with $\mathrm{n}=$ number of $\mathrm{C}$ atoms) incorporated in junctions having structure $\mathrm{Ag}^{\mathrm{TS}}-\mathrm{SAM} / / \mathrm{Ga}_{2} \mathrm{O}_{3} / \mathrm{EGaIn}$, data for tunneling rates for the range of lengths $n=1-18$ lead to a value for the injection tunnel current density $J_{o}$ (i.e., the current flowing through an ideal junction with $\mathrm{n}=0)$ of $J_{o}=10^{3.6 \pm 0.3} \mathrm{~A} \cdot \mathrm{cm}^{-2}(\mathrm{~V}=+0.5 \mathrm{~V})$. This estimation of $J_{o}$ does not involve an extrapolation in length, because it was possible to measure current densities across SAMs over the full range $\left(n=1\right.$ to $n=18$ ) of lengths. The electrical resistance of the $\mathrm{Ga}_{2} \mathrm{O}_{3}$ layer is 10 times less than the resistance of the shortest $\mathrm{SAM}\left(\mathrm{SC}_{1}\right)$ and does not contribute to $J_{o}$. This value of $J_{o}$, however, is estimated under the assumption that values of the geometrical contact area $\left(\mathrm{A}_{\mathrm{geo}}\right)$ equal the values of the effective electrical contact area. Detailed experimental analysis indicates that the roughness of the $\mathrm{Ga}_{2} \mathrm{O}_{3}$ layer, and of the $\mathrm{Ag}^{\mathrm{TS}}-\mathrm{SAM}$, determine values of the effective electrical contact area that are $\sim 10^{-4}$ the corresponding values of the geometrical contact area; the conversion of the values of geometrical contact area $\left(\mathrm{A}_{\text {geo }}\right)$ into the corresponding values of effective electrical contact area $\left(10^{-4.0 \pm 0.5} \cdot \mathrm{A}_{\mathrm{geo}}\right)$ results in a estimated, corrected value of the effective injection current density of $J_{o}(+0.5 \mathrm{~V})=10^{7.6 \pm 0.8} \mathrm{~A} \cdot \mathrm{cm}^{-2}$; this value is compatible with values reported for junctions using top-electrodes of evaporated $\mathrm{Au}$, and graphene, and also comparable with values of $J_{o}$ estimated from tunneling through single molecules in junctions using STM and AFM tips. The value of the tunneling decay factor $\beta$ for SAMs of $n$-alkanethiolates $\left(\beta=0.75 \pm 0.02 \AA^{-1} ; \beta=0.92 \pm 0.02 \mathrm{nC}^{-1}\right)$ estimated in junctions using $\mathrm{Ga}_{2} \mathrm{O}_{3} /$ EGaIn conical tips falls within the consensus range across different types of junctions $\left(\beta=0.73-089 \AA^{-1} ; \beta=0.9-1.1 \mathrm{nC}^{-1}\right)$. A comparison of the characteristics of conical $\mathrm{Ga}_{2} \mathrm{O}_{3} / \mathrm{EGaIn}$ tips with other top-electrodes suggests that the EGaIn-based electrodes provide a particularly attractive technology for physical-organic studies of charge transport across SAMs. 


\section{Introduction}

Measurements, using a number of techniques, of rates of charge transport by tunneling across self-assembled monolayers (SAMs) of $n$-alkanethiolates on silver and gold substrates show an interesting, puzzling, and unresolved mixture of consistency and inconsistency. Rates of tunneling across these SAMs follow the simplified Simmons equation ${ }^{1,2}$ (Eq. 1), $J(V)=J_{0}(V) \cdot 10^{-\beta \cdot d / 2.303}$

with the fall-off in current density $J(\mathrm{~V})\left(\mathrm{A} \cdot \mathrm{cm}^{-2}\right)$ with increasing length $d$ of the $n$-alkyl group giving (for even-numbered carbon chains, and at voltages in the range $\mathrm{V}= \pm 0.5 \mathrm{~V}$ ) approximately the same value of $\beta$ by most or all methods of measurement ( $\beta=0.73-0.89 \AA^{-1}$; for $d=\mathrm{nC}=$ number of carbon atoms, $\left.\beta=0.90-1.1 \mathrm{nC}^{-1}\right)$. Using $\mathrm{Hg}$-drops as top-electrodes, measurements of rates of tunneling across $n$-alkanes anchored to heavily doped silicon surfaces led to a value of $\beta\left(\beta=0.9 \pm 0.2 \mathrm{nC}^{-1}\right)$ similar to those observed for $n$-alkanethiolates on $\mathrm{Au}$ and Ag substrates ${ }^{3,4}$

By contrast, values of the injection current $J_{o}(\mathrm{~V}=+0.5 \mathrm{~V})$ - the limiting value of current for an ideal junction with no hydrocarbon present $(d=0)$, but with all the interfaces and characteristics of junctions containing the SAMs — vary from $\sim 10^{8} \mathrm{~A} \cdot \mathrm{cm}^{-2}$ estimated from single-molecules approaches ${ }^{5-10}$, and measured for graphene ${ }^{11}$ and evaporated gold ${ }^{12}$ topelectrodes, to $\sim 10^{2} \mathrm{~A} \cdot \mathrm{cm}^{-2}$ observed in large-area junctions using, as top-electrodes, conductive polymers $^{13}$, mercury drops supporting an insulating organic film $(\mathrm{Hg}-\mathrm{SAM})^{14-16}$, and $\mathrm{Ga}_{2} \mathrm{O}_{3} / \mathrm{EGaIn}$ tips $^{17-20}$. Why is there high consistency in values of $\beta$, but broad inconsistency in values of $J_{o}(\mathrm{~V})$ within these systems?

A priori, at least four factors might contribute to differences in $J_{o}(\mathrm{~V})$ among methods of measurements: i) In large-area junctions, assuming that the effective electrical contact area 
$\left(\mathrm{A}_{\text {elec }}\right)$ - the area through which current actually passes - coincides with the geometrical contact area $\left(\mathrm{A}_{\mathrm{geo}}\right)$ estimated by optical microscopy could result in errors in the conversions of values of current into current densities. Contact between surfaces occurs only through asperities distributed on the surfaces, which are always rough to some extent; in addition, only a fraction of the true, physical contact is conductive $\mathrm{e}^{21-24}$. Estimations of the effective contact area from measurements of adhesion and friction between surfaces indicate that values of $A_{\text {eled }} / A_{\text {geo }}$ vary in the range $10^{-2}$ $10^{-4}$, depending on the hardness of the materials, the heights, widths, and number of asperities on both surfaces, and loads applied to the contacts ${ }^{22,23,25-27}$. ii) The resistivities of the $\mathrm{SAM} / /$ electrode contacts might vary due to the nature of the interactions at the top-interface (e.g., covalent bonding versus van der Waals contacts), to differences in the resistivities of the electrode materials, and to the presence of adsorbed insulating impurities. iii) The preparation of the top-electrode might damage the SAM (e.g., by reaction of hot metal atoms condensing on top of the SAM, by formation of metal filaments that partially or completely bridge the $\mathrm{SAM}^{28-30}$, and/or by displacing the SAM). iv) The range may also, to some extent, be an artifact: values of $J_{o}$ have often been determined by long extrapolations from small ranges of lengths, and based on data characterized by large (and often not statistically determined) dispersions in measured values of current densities for individual $n$-alkanethiolates.

There has been much speculation about the relative importance of these factors, but little experimental evidence with which to decide among them. Review articles ${ }^{31,32}$ have considered (approximately) that the electrical behavior of different types of junctions might be influenced by the bulk resistivities of the materials used for the top-electrodes. We observe, however, that the correlation of the structures of the molecules making up the SAM with rates of charge transport through these molecules would be impossible if the electrical properties of the top-electrode 
were to dominate the behavior of the junctions. By contrast, the consensus on the value of $\beta$ for tunneling across SAM of $n$-alkanethiolate $\left(\beta=0.73-0.89 \AA^{-1} ; \beta=0.9-1.1 \mathrm{nC}^{-1}\right)$ strongly suggests that these junctions capture details that are characteristic of charge transport through molecules. $\mathrm{We}^{17,19,20,33-37}$ and others ${ }^{38-40}$ have been developing $\mathrm{Ga}_{2} \mathrm{O}_{3} / \mathrm{EGaIn}$ soft top-electrodes to contact SAMs formed on template-stripped Au and Ag substrates. We have previously reported that the layer (average thickness of $\sim 0.7 \mathrm{~nm}$ in simple, mechanically unstressed systems) that spontaneously forms on the surface of EGaIn-electrodes consists primarily of $\mathrm{Ga}_{2} \mathrm{O}_{3}{ }^{41}$, and that this $\mathrm{Ga}_{2} \mathrm{O}_{3}$ layer is electrochemically inactive in the range $\mathrm{V}= \pm 1 \mathrm{~V}^{34,42}$. Two of the remaining areas that still need better definition are the value of the effective area of electrical contact of the SAM with the $\mathrm{Ga}_{2} \mathrm{O}_{3} /$ EGaIn top-electrode, and the resistance of the $\mathrm{Ga}_{2} \mathrm{O}_{3}$.

This paper reaches five major conclusions: i) In junctions using conical $\mathrm{Ga}_{2} \mathrm{O}_{3} / \mathrm{EGaIn}$ electrodes on top of SAMs formed on template-stripped silver substrates, the effective electrical contact area is $\sim 10^{-4}$ the geometrical contact area (measured by optical microscopy). ii) The resistance of the $\mathrm{Ga}_{2} \mathrm{O}_{3}$ layer is lower than the resistance of the SAM of the shortest $n$-alkanethiolate $\left(\mathrm{Ag}^{\mathrm{TS}}-\mathrm{S}-\mathrm{CH}_{3}\right)$ and makes no significant contribution to the resistance of the junction for any length of the $n$-alkanethiolate group (Figure 1). iii) Flattening and stabilizing the surface of the $\mathrm{Ga}_{2} \mathrm{O}_{3} / \mathrm{EGaIn}$ tip reduces (by $\sim 60 \%$ ) the dispersion in values of $\log |J(\mathrm{~V})|$. iv) For junctions having the structure $\mathrm{Ag}^{\mathrm{TS}}-\mathrm{SR} / / \mathrm{Ga}_{2} \mathrm{O}_{3} / \mathrm{EGaIn}\left(\mathrm{Ag}^{\mathrm{TS}}=\right.$ template-stripped silver substrate; $\mathrm{R}=\mathrm{C}_{\mathrm{n}} \mathrm{H}_{2 \mathrm{n}+1}$, with $\left.\mathrm{n}=0-18\right), J_{o}=10^{3.6 \pm 0.3} \mathrm{~A} \cdot \mathrm{cm}^{-2}$ at $\mathrm{V}=+0.5 \mathrm{~V}$. This value of $J_{o}$ is much more accurate than previous estimates, in part because we can measure tunneling currents through short-chain ( $n=1-4)$ alkyl groups. v) Approximating the effective electrical contact area by the geometrical contact area leads to a significant overestimation of the electrical contact area and to an underestimation of the effective value of $J_{o}$. Correcting the value of $J_{o}$ determined 
experimentally by the estimated ratio $\mathrm{A}_{\text {eled }} / \mathrm{A}_{\text {geo }}=10^{-4.0 \pm 0.5}$ gives $J_{o}=10^{7.6 \pm 0.8} \mathrm{~A} \cdot \mathrm{cm}^{-2}(\mathrm{~V}=+0.5 \mathrm{~V})$; this correction reconciles the value of $J_{o}$ for $\mathrm{Ag}^{\mathrm{TS}}-\mathrm{SAM} / / \mathrm{Ga}_{2} \mathrm{O}_{3} / \mathrm{EGaIn}$ junctions with the values of $J_{o}$ reported for single molecules approaches ${ }^{5-9}$, graphene ${ }^{11}$, and evaporated gold ${ }^{12}$ electrodes $\left(10^{8}-10^{9} \mathrm{~A} \cdot \mathrm{cm}^{2}\right)$

We emphasize that the correction of the electrical contact area has no influence on appropriately designed physical-organic studies, since these studies rely entirely on comparisons of tunneling currents across different organic groups for which the $\mathrm{Ag}^{\mathrm{TS}}-\mathrm{SR}$ and the $\mathrm{SAM} / / \mathrm{Ga}_{2} \mathrm{O}_{3}$ interfaces will have the same properties; top-interfaces involving SAMs formed with different types of molecules, and with different electronic structures (e.g., $\mathrm{Ag}^{\mathrm{TS}}$-S-alkyl, and $\mathrm{Ag}^{\mathrm{TS}}$-S-aromatic), may be different for various reasons. In this work, we thus assume that the correction of the values of current density $J(V)$ for the effective electrical contact area is unnecessary for physical-organic studies comparing SAMs derived from $\mathrm{HSC}_{\mathrm{n}}$. For this type of studies, our estimation of $J_{o}$-a value that is particularly accurate because it does not involve the long extrapolation from $n=10-18$ to $n=0$ characteristic of most prior works - provides a reference value that can be used to test the quality of data for tunneling across $n$-alkanethiolates collected (using conical $\mathrm{Ga}_{2} \mathrm{O}_{3} / \mathrm{EGaIn}$ tips) in different laboratories.

\section{Background}

$\mathrm{Ga}_{2} \mathrm{O}_{3} / \mathbf{E G a I n}$ as a Material for Top-Electrodes. We are developing liquid top-electrodes of eutectic gallium indium alloy (EGaIn) for studies of charge transport through SAMs. Upon exposure to air, EGaIn forms (essentially instantaneously on the time scale of these experiments) a thin (nominally $\sim 0.7 \mathrm{~nm}^{40}$ ), self-passivating oxide layer (mostly $\left.\mathrm{Ga}_{2} \mathrm{O}_{3}\right)^{41,43}$. This layer, although mechanically fragile, enables us to fabricate geometrically defined tips ${ }^{44}$. During 
fabrication, and in contacting the SAM, it buckles, and this buckling generates rough $\mathrm{Ga}_{2} \mathrm{O}_{3}$ surfaces.

For most of our work, we have used $\mathrm{Ga}_{2} \mathrm{O}_{3} / E$ EaIn conical tips as top-electrodes for five reasons: i) They can (partially) adapt to the topography of the substrate. ii) They are particularly convenient to use in physical-organic studies, which require trends in $J(\mathrm{~V})$ with the structure of the SAM rather than absolute values of $J(V)$. iii) They appear not to damage the SAM. vi) They do not require expensive hardware or sophisticated equipment to generate reproducible data for current density. v) They make it possible to collect large numbers of data $\left(\sim 10^{3}\right.$ complete $J$-V sweeps per day); these numbers provide the basis for detailed statistical analyses of uncertainty, dispersion, and variability of values. We point out that many of the results for molecular tunneling described in the earlier literature appear to rely on single, selected data points, or small numbers of points, and are literally uninterpretable, because they do not distinguish between statistically defined values (means based on large number of data), and outliers or artifacts (which may be displaced many orders of magnitude from the mean) ${ }^{18,45}$.

Electrical Structure of the $\mathrm{Ag}^{\mathrm{TS}}-\mathrm{SAM} / / \mathrm{Ga}_{2} \mathrm{O}_{3} / \mathbf{E G a I n}$ Junction. For a fixed voltage, we can consider junctions having structure $\mathrm{Ag}^{\mathrm{TS}}-\mathrm{SAM} / / \mathrm{Ga}_{2} \mathrm{O}_{3} / \mathrm{EGaIn}$ as being two resistors in series (Figure 2): the $\mathrm{Ga}_{2} \mathrm{O}_{3}$ layer (with specific resistance $R_{\mathrm{Ga}_{2} \mathrm{O}_{3}}$ ), and the tunnel gap established by the SAM. This tunnel gap, which has specific resistance $R_{S A M}$, extends from the surface of the $\mathrm{Ag}^{\mathrm{TS}}$ bottom-substrate to the surface of the $\mathrm{Ga}_{2} \mathrm{O}_{3}$ layer of the top-electrode. The resistance of the junction is thus given by (Eq. 2):

$R_{J}=R_{S A M}+R_{\mathrm{Ga}_{2} \mathrm{O}_{3}}$

The tunneling resistance $R_{\text {SAM }}$ originates, in principle, from three components (Figure 2): 1) the resistance of the van der Waals top-interface, which has thickness $l_{v d W}$. ii) The resistance 
of tunnel barrier established by the alkyl chain. For a through-molecule transport, the length $d$ of this tunnel barrier is given by the length of the alkyl chain. 3) The resistance of the Ag-S covalent contact, whose length is $l_{A g-S}$. Adopting, for these three components, the formalism of the Simmons model, the resistance of the tunnel gap can be (formally) written as (Eq. 3): $R_{S A M}=R_{o}^{o} \cdot e^{-\beta_{v d W} \cdot l_{v d W}} \cdot e^{-\beta_{A g-S} \cdot l_{A g-S}} \cdot e^{-\beta \cdot d}$ Eq. 3

In Eq. 3, $R_{o}^{o}$ accounts for the electronic properties of the surfaces of the $\mathrm{Ag}^{\mathrm{TS}}$ substrate and of the $\mathrm{Ga}_{2} \mathrm{O}_{3}$ layer; $\beta_{\mathrm{Ag}-\mathrm{S}}$ and $\beta_{v d W}$ are the hypothetical decay factors for tunneling across the Ag-S and the $\mathrm{SAM} / / \mathrm{Ga}_{2} \mathrm{O}_{3}$ van der Waals interface; and $\beta$ is the tunneling decay factor characteristic of $n$-alkanes.

If $R_{\mathrm{Ga}_{2} \mathrm{O}_{3}}<<R_{\mathrm{SAM}}$, the electrical behavior of the junction is dominated by tunneling across the SAM ( $\left.R_{J} \approx R_{S A M}\right)$, and trends in $J(\mathrm{~V})$ with the structure of the molecules in the SAM will be useful in understanding the relationship between molecular structures and tunneling rates. If, however, the resistance of the $\mathrm{Ga}_{2} \mathrm{O}_{3}$ were similar $\left(R_{\mathrm{Ga}_{2} \mathrm{O}_{3}} \approx R_{\mathrm{SAM}}\right)$, or larger $\left(R_{\mathrm{Ga}_{2} \mathrm{O}_{3}}>R_{\mathrm{SAM}}\right)$ than that of the SAM, interpretation of data for charge transport through SAMs could be difficult or impossible.

In a previous study ${ }^{34}$, our group estimated a value of the specific resistance (i.e., the resistance per unit area) of the $\mathrm{Ga}_{2} \mathrm{O}_{3}$ layer of $R_{\mathrm{Ga}_{2} \mathrm{O}_{3}} \sim 4 \times 10^{-2} \Omega \cdot \mathrm{cm}^{2}$. This value of $R_{\mathrm{Ga}_{2} \mathrm{O}_{3}}$ was $\sim 10^{4}$ times lower than that of a SAM composed of $\mathrm{SC}_{9}$, the shortest molecule studied at that time $^{19}$. We thus concluded that, for medium-length SAMs (n=9-18), the values of $J(V)$ were determined by the SAM; this inference did not automatically apply to short-chain SAMs (which were not examined in previous studies). In this work, we correct this value to $R_{\mathrm{Ga}_{2} \mathrm{O}_{3}}=3.3 \times 10^{-4}$ $\Omega \cdot \mathrm{cm}^{2}$; this change does not alter our understanding of the junction. 
Properties of the SAM//Ga $\mathrm{O}_{3} /$ EGaIn Top-Contact, and Sources of Uncertainty. The $\mathrm{Ga}_{2} \mathrm{O}_{3} / \mathrm{EGaIn}$ top-electrode is in van der Waals contact with SAMs of $n$-alkanethiolates, which terminate in $\mathrm{CH}_{3}$ groups. Because the formation and manipulation of conical tips generate a $\mathrm{Ga}_{2} \mathrm{O}_{3}$ layer that is variable in thickness, and that further buckles and unfolds during manipulation, the surface of the top-electrode is rough. The surface of the $\mathrm{Ag}^{\mathrm{TS}}$ substrate, and thus the topography of the top-surface of the SAM, is also rough (due to steps, grain boundaries, and other defects in the silver surface, and to irregularities in the order and structure of the $\mathrm{SAM}^{46}$ ). This heterogeneity in structure, topography, and thickness results in an effective electrical contact area that is smaller than the area of the geometrical contact estimated by microscopy.

In addition to the heterogeneities in the $\mathrm{Ga}_{2} \mathrm{O}_{3}$ film, other factors that can contribute to uncertainties in data collected with $\mathrm{Ga}_{2} \mathrm{O}_{3} / \mathrm{EGaIn}$ top-electrodes include (i) the presence of adventitious contaminants adsorbed on either the tip and/or the SAM (although a methylterminated surface has a low interfacial free energy and a low tendency to adsorb impurities, metals and metal oxides have high surface energies); and (ii) the formation of conductive filaments between the top-electrode and the metal substrate ${ }^{28-30}$.

Charge Transport through SAMs. The Simmons model ${ }^{1,2}$ provides an approximate analytical equation (Eq. 1) for charge tunneling through a potential barrier established by an organic insulator; in this model, the value of the decay factor $\beta$ is determined by the height of the potential barrier. Currently, no molecular theory correlates experimental values of $J(\mathrm{~V})$ and $\beta$ with molecular structure, and the Simmons model may be inappropriate and incomplete ${ }^{47,48}$ (especially for organic SAMs more complicated than simple $n$-alkanes, or when these SAMs involve electronically conductive regions, although we observed that charge transport through a 
variety of complex structures is very similar to tunneling through $n$-alkanethiolates ${ }^{37}$ ). Nonetheless, in the absence of a more developed theory, the simplified Simmons equation has been adopted as the theoretical standard for analyses in studies of charge tunneling through organic molecules, because, in the low bias regime, it reduces the tunneling problem to the determination of just two parameters: $\beta$ and $J_{o}$.

Values of $J_{o}$ for tunneling through $n$-alkanethiolates estimated by different techniques differ by more than $10^{7} \mathrm{~A} \cdot \mathrm{cm}^{-2}$; these techniques use a variety of different top-electrodes, which include $\mathrm{Hg}$-drops ${ }^{49}, \mathrm{Hg}-\mathrm{SC}_{\mathrm{n}} \mathrm{SCH}_{3}{ }^{14-16,50}$ (that is, $\mathrm{Hg}$ drops supporting a monolayer of $\mathrm{S}\left(\mathrm{CH}_{2}\right)_{\mathrm{n}} \mathrm{CH}_{3}$, with $\left.\mathrm{n}=11,13,15\right)$, conductive polymers ${ }^{13,47}, \mathrm{Ga}_{2} \mathrm{O}_{3} / \mathrm{EGaIn}^{18-20}$ tips, evaporated $\mathrm{Au}$ electrodes ${ }^{12}$, Au electrodes fabricated via nanoskiving ${ }^{51}$, graphene ${ }^{11}$, and $\mathrm{STM}^{5-7}$ and $\mathrm{AFM}^{8,9}$ tips. Table 1 summarizes values of $J_{o}(\mathrm{~V}=+0.5 \mathrm{~V})$ reported or estimated for these junctions.

Experiments with different junctions using the same material for the top-electrode have given substantial differences in $J_{o}$ : this variability is in contradiction with theory and intuition, both of which would predict that same materials would give same values of $J_{o}(\mathrm{~V})$. Our hypothesis is that differences in $J_{o}$ arise from differences in the procedures used to fabricate the top-electrode, rather than from differences in materials. Junctions using Au electrodes directly evaporated ${ }^{12}$ on top of the SAM yielded a value of $J_{o}\left(10^{8} \mathrm{~A} \cdot \mathrm{cm}^{-2}\right)$ that was $10^{5}$ that reported for junctions using Au top-electrodes fabricated via nanoskiving ${ }^{51}\left(J_{o}=10^{3.2} \mathrm{~A} \cdot \mathrm{cm}^{-2}\right)$. We believe both values are correct, but specific to different experiments: the different behavior probably reflects differences in the value of $\mathrm{A}_{\text {elec }} / \mathrm{A}_{\text {geo }}$ due to differences in the roughness of the electrodes.

Using highly conductive polyphenylenevinylene layers ${ }^{13}$, and Hg-drops carrying an organic insulator ${ }^{15}$ as top-contacts on SAMs formed on rough (as-evaporated) substrates yielded values of $J_{o}\left(10^{2}-10^{3} \mathrm{~A} \cdot \mathrm{cm}^{-2}\right)$ close to the values of $J_{o}$ obtained using highly conductive, but 
rough $\mathrm{Ga}_{2} \mathrm{O}_{3} / E$ EaIn conical tips ${ }^{18,20}$. By contrast, values of $J_{o} \sim 10^{8} \mathrm{~A} \cdot \mathrm{cm}^{-2}$ are reported for largearea junctions using flat metal substrates and flat (e.g., graphene $\left.{ }^{11}\right)$, or compliant (e.g., evaporated $\left.\mathrm{Au}^{12}\right)$ top-electrodes; these values of $J_{o}(\mathrm{~V})$ are close to those estimated for singlemolecules techniques $\left(\sim 10^{8}-10^{9} \mathrm{~A} \cdot \mathrm{cm}^{-2}\right)$.

For junctions formed with $\mathrm{Hg}$-drops on top of alkyl chains anchored to heavily doped $\mathrm{Si}$ surfaces, Cahen and co-workers reported a value of $J_{o} \sim 10^{6.5} \mathrm{~A} \cdot \mathrm{cm}^{-2}$ for $\mathrm{p}$-Si substrates ${ }^{4}$, and a value of $J_{o} \sim 10^{4} \mathrm{~A} \cdot \mathrm{cm}^{-2}$ for $\mathrm{n}$-Si substrates ${ }^{48}$ (we estimated these values of $J_{o}$ from data reported by the authors ${ }^{4,48}$ ). For the system with $J_{o} \sim 10^{4} \mathrm{~A} \cdot \mathrm{cm}^{-2}$, however, fitting the Simmons equation to experimental $J-\mathrm{V}$ curves for individual $n$-alkanes required a correction of the contact area by a factor of $\sim 10^{-4}$ in order to get meaningful fitting parameters ${ }^{3}\left(30 \mu \mathrm{m}^{2}\right.$ contact area required by the fitting, over a geometrical contact area of $5 \times 10^{4} \mu \mathrm{m}^{2}$ ); Cahen et al. argued that theoretical modeling of experimental tunneling rates characterized by $J_{o} \leq 10^{4} \mathrm{~A} \cdot \mathrm{cm}^{-2}$ "may lack physical relevance",

These results indicate that different properties (we believe primarily topographies of the surfaces) of top- and bottom- electrodes — and not the resistances of the corresponding bulk materials - determine the spread of the experimental values of $J_{o}$ across different methods (Table $1)$.

\section{Experimental design}

SAMs of $\boldsymbol{n}$-Alkanethiolates with Even Number of Carbon Atoms. $n$-Alkanethiols $\left(\mathrm{CH}_{3}\left(\mathrm{CH}_{2}\right)_{\mathrm{n}-1} \mathrm{SH}\right.$, represented here as $\left.\mathrm{SC}_{\mathrm{n}}\right)$ are commercially available in a range of lengths; they are accepted as the simplest model system for physical-organic studies of tunneling through SAMs (we note that the complexity of structural features of SAMs of $n$-alkyl chains- e.g., packing density; insoluble multilayer metal thiolate "soaps" for silver; defects; roughness of the 
underlying metal—all make these SAMs more complex than they first seem ${ }^{52}$ ). We collected $J$-V data for SAMs composed of $\mathrm{SC}_{\mathrm{n}}$ with even numbers of carbons from $\mathrm{n}=2$ to $\mathrm{n}=18$. We excluded long ( $>5$ ) $n$-alkanethiolates with odd numbers of carbons because they behave differently from those with even $n$, and require a separate analysis ${ }^{14,19}$.

\section{Measurements on Short n-Alkanethiolates with Odd Numbers of Carbon Atoms. In} order to study the frequency of the failure of the junctions, and to provide values of $J(\mathrm{~V})$ for SAMs composed of short molecules, we measured rates of charge transport through SAMs formed from $\mathrm{CH}_{3} \mathrm{SH}\left(\mathrm{SC}_{1}\right)$, and $\mathrm{CH}_{3}\left(\mathrm{CH}_{2}\right)_{2} \mathrm{SH}\left(\mathrm{SC}_{3}\right)$, and through $\mathrm{Ag}^{\mathrm{TS}}-\mathrm{SH}\left(\mathrm{SC}_{0}\right)$. (We formed $\mathrm{CH}_{3} \mathrm{SH}$, and $\mathrm{H}_{2} \mathrm{~S}$ in situ by hydrolysis of $\mathrm{CH}_{3} \mathrm{SNa}$, and $\mathrm{Na}_{2} \mathrm{~S}$ respectively). We describe experimental details of the preparation of the SAMs in the Supporting Informations.

Template Stripped Substrates. We formed SAMs on template-stripped $\mathrm{Ag}\left(\mathrm{Ag}^{\mathrm{TS}}\right)$ substrates ${ }^{19,46}$. Template stripping provides surfaces characterized by large flat terraces (average area of terraces $\sim 0.13 \mu \mathrm{m}^{2}$ ). Template-stripped surfaces give larger effective electrical contact areas than "as-evaporated" top-surfaces ${ }^{46}$; the value of $\mathrm{A}_{\text {eled }} / \mathrm{A}_{\text {geo }}$ is, however, limited by the roughness of the terraces, by the size of the silver grains, and by the width and the depth of groves between grains. Forming SAMs on silver template-stripped substrates yielded $\sim 90 \%$ of non-shorting junctions.

\section{Fabrication of Flattened Conical Tips, and Formation of Stable Contacts on Top of}

the SAM. The apparent non-Newtonian properties of the $\mathrm{Ga}_{2} \mathrm{O}_{3} / \mathrm{EGaIn}^{43}$ enable several procedures for the fabrication of the top-electrode. For this paper we used what we call "flattened" conical tips. Although these tips are characterized by an effective electrical contact area that we know to be less than the nominal contact area we measure by optical microscopy, they can be quickly and easily fabricated, and used in physical-organic studies, where 
convenience and the ability to generate large number of data are more important in correlating different molecular systems with values of $J(\mathrm{~V})$ than is high precision (this type of use assumes, of course, that $J_{o}(\mathrm{~V})$ is the same across all the junctions formed with different tips). Figure 3 shows the five steps of the procedure we used to fabricate flattened conical tips: (1) We extruded a $\mathrm{Ga}_{2} \mathrm{O}_{3}$ EGaIn drop from a $10-\mu \mathrm{L}$ syringe (In order to keep the weight of the EGaIn column constant in all junctions, we always used $2 \mu \mathrm{L}$ of EGaIn). We formed conical tips only after a minimum of three hours from the time of the refilling of the syringe with fresh EGaIn. (Currents measured with tip fabricated immediately after refilling the syringe show large variability, or fluctuating values.) (2) With a micro-manipulator, we brought the $\mathrm{Ga}_{2} \mathrm{O}_{3} / \mathrm{EGaIn}$ drop in contact with a clean metal substrate (usually $\mathrm{Au}^{\mathrm{TS}}$ ). (3) We slowly $(\sim 0.5 \mathrm{~mm} / \mathrm{sec})$ pulled the syringe away from the surface to form a $\mathrm{Ga}_{2} \mathrm{O}_{3} / \mathrm{EGaIn}$ neck. (4) By further withdrawing the syringe, we broke the $\mathrm{Ga}_{2} \mathrm{O}_{3} / \mathrm{EGaIn}$ neck and obtained a top-conical tip. The surface of this as-fabricated conical tip is characterized by asperities and whiskers randomly formed while forming and breaking the neck. Analysis of data collected with these tips revealed that an important source of dispersion in the measured values of $J(\mathrm{~V})$ was the variation of these values from junction to junction, probably due to the progressive deformation of asperities (Supporting Information Figure S1). (5) We flattened the asperities of the as-fabricated conical tip by touching it three times to a flat, polished $\mathrm{SiO}_{2} / \mathrm{Si}$ surface (the type of $\mathrm{SiO}_{2} / \mathrm{Si}$ substrates used for template-stripped metal surfaces). We observed that this procedure stiffened the tip, and led to more stable contacts. We also observed that running three cyclic voltage scans between $\pm 2 \mathrm{~V}$ while the tip is contact with the Si chip further reduced the dispersion in data for $J$.

We used the flattened conical tips as top-electrodes on $\mathrm{Ag}^{\mathrm{TS}}$-SAM substrates (Figure 3, step 6). To form stable contacts, we brought the tips closer to the substrates until we 
could clearly observe (by optical microscopy) the tip apex wrinkling, or deforming; from this point, current densities did not change by further pushing the tips against the substrate. (We thus used the "wrinkling" of the $\mathrm{Ga}_{2} \mathrm{O}_{3}$ skin on the sides of the conical tip as an indication of the load applied). We did not explore large deformation of the tips.

This procedure for the fabrication of flattened tips, and for the formation of stable contacts on top of the SAMs generated reproducible values of $J_{o}(\mathrm{~V}), \beta$ and $\sigma_{\log }$ among different users. The standard deviations of values of $\log |J|$ measured with these flattened conical tips were smaller by $60 \%$ smaller than those observed for as-fabricated tips; flattening the tip, however, had no effect on the mean values $<\log |J|>$ of the distributions (Figure S1 Supporting Information). A video of the fabrication of a flattened conical tip is available as supporting material.

Protocol for Collection of Data. We have observed that data collected with an unstandardized protocol (that is, forming an arbitrary number of junctions, running arbitrary numbers of scans) can broaden data distributions. We have thus introduced a standard procedure for data acquisition, which we call the " $1 / 3 / 20$ " protocol. For each individual tip, we formed three junctions (i.e., contacts) in three different places of the substrate (that is, the SAM supported on $\mathrm{Ag}^{\mathrm{TS}}$ ); for each junction, we recorded $20 \mathrm{~J}-\mathrm{V}$ traces for forward bias $(\mathrm{V}=-0.5 \mathrm{~V} \rightarrow \mathrm{V}=+0.5 \mathrm{~V})$ and $20 \mathrm{~J}(\mathrm{~V})$ traces for reverse bias $(\mathrm{V}=+0.5 \mathrm{~V} \rightarrow \mathrm{V}=-0.5 \mathrm{~V})$. From empirical comparison of procedures, we speculate that, by limiting the number of junctions per tip, the $1 / 3 / 20$ protocol may avoid collecting data with EGaIn tips contaminated by adventitious impurities. The twenty scans per junction provide enough information about the variation of $J$ within the same junction to determine standard deviations. Collecting the same number of data points per tip (120 points in total) ensures that all tips have the same statistical weight. 
Definition of the Tunnel Injection Current Density $J_{o}(V)$. In this paper, we define the injection current $J_{o}(\mathrm{~V})$ for $\mathrm{Ag}^{\mathrm{TS}}-\mathrm{SC}_{\mathrm{n}} / / \mathrm{Ga}_{2} \mathrm{O}_{3} / \mathrm{EGaIn}$ junctions as the value of $J(\mathrm{~V})$ given by Eq. 3 for $d=0$, that is, the value of $J(\mathrm{~V})$ for an ideal junction with no alkyl chain, but with all characteristics and interfaces of junctions containing the SAM (Eq. 4):

$$
J_{o}(V)=V /\left(R_{o}^{o} \cdot e^{-\beta_{V d W} \cdot d_{V d W}} \cdot e^{-\beta_{A g-s} \cdot d_{A g-S}}\right) \equiv V / R_{o}
$$

We adopted this definition of $J_{o}(\mathrm{~V})$ for two reasons: 1) The value of $J_{o}(\mathrm{~V})$ given by Eq. 4 can be easily estimated by extrapolating to $d=0$ values of $\log |J(\mathrm{~V})|$ for $n$-alkanethiolates of different lengths. The extrapolation requires that values of $\log |J(\mathrm{~V})|$ decay with a constant slope (that is, constant $\beta$ ) along the full range of lengths. We recall that the tunnel barrier includes also the Ag-S and SAM $/ / \mathrm{Ga}_{2} \mathrm{O}_{3}$ van der Waals interfaces. But the $\beta$ factors of the top- and bottominterfaces are unknown; assuming that the interfaces have the same $\beta$ as the alkyl chain might lead to errors in the estimation of the value of $J_{o}$ (this error might be significant for SAMs formed using anchoring group longer than $\mathrm{Ag}-\mathrm{S}$, as, for example, $\mathrm{C} \equiv \mathrm{C}-\mathrm{R}$, or $\mathrm{O}_{2} \mathrm{C}-\mathrm{R}^{53}$ ). 2) Defining $J_{o}(\mathrm{~V})$ for $d=0$ gives a value of injection current that incorporates the rates for charge transport across the top- and bottom-interface (Eq. 4). This value of $J_{o}(\mathrm{~V})$ can be used to compare the efficiency of charge injection of thiolates with that of other anchoring groups (provided that for these other anchoring groups $d=0$ is defined on the first atom to which the alkyl chain is bound, and that the van der Waals top-interface remains unchanged); alternatively, values of $J_{o}(\mathrm{~V})$ for SAMs of $n$-alkyl-thiolates having different terminal groups can be used to compare the efficiencies of charge injections into van der Waals top-interfaces with different structures.

We point out that the value of $J_{o}$ given by Eq. 4 varies linearly with the applied voltage (that is, constant value of $R_{o}$ ) only in the range of voltages $-0.1 \mathrm{~V} \leq \mathrm{V} \leq+0.1 \mathrm{~V}^{20}$. Assuming, 
however, that the value of $R_{o}$ is invariant, for a fixed voltage, across SAMs of different $n$ alkanethiolates, in this paper we estimate the value of $J_{o}$ for the applied voltage $\mathrm{V}=+0.5 \mathrm{~V}$ (and $\mathrm{V}=-0.5$ ). At $\mathrm{V}=+0.5 \mathrm{~V}$, rates of charge tunneling follows (qualitatively) the Simmons equation (Eq. 1); in addition, values of tunneling current across SAMs of the full set of $n$-alkanethiolates $\left(\mathrm{SC}_{\mathrm{n}}\right.$, with $\left.\mathrm{n}=1-18\right)$ fell within the operative range of our electrometer $(105 \mathrm{~mA}-0.1 \mathrm{nA})$. At voltages $>+0.5 \mathrm{~V}(<-0.5 \mathrm{~V})$, resonances of the Fermi levels of the electrodes with molecular or interfacial electronic states lead to non-linear variation of $J_{o}$ with $\mathrm{V}^{54}$, and the approximate Simmons equation does not describe charge tunneling adequately. In this paper we do not explore the high-bias regime.

Assuming a through-molecule tunneling, we measure the thickness $d$ of the tunnel barrier established by the $n$-alkyl chains as the distance in $\AA$ from the S-terminus of the $n$-alknethiolates to the distal hydrogen atom closest to the top-electrode (Figure 2). To compare our results with others reported in the literature, we also estimated $d$ as the number $(\mathrm{nC})$ of $\mathrm{C}$ atoms of the alkyl backbone; we observe, however, that the length of the molecules as $\mathrm{nC}$ does not take into account the contribution of the length of the distal C-H bond(s) to the width of the tunneling barrier generated by trans-extended $n$-alkanethiolates (Figure S2), and thus leads to a systematic underestimation of the extrapolated value of $J_{o}$. In addition, giving $d$ as $\mathrm{nC}$ might also lead to apparent inconsistencies between the electrical behavior of alkyl chains with odd and even number of $\mathrm{C}$, because, due to the tilt angle of the SAM, the orientation of the distal C-H bond (and thus the effective length of the tunnel barrier) differs for these two classes of $n$ alknethiolates (Figure S2). 
It is evident, however, that the comparison of the electrical behavior of SAMs of $n$ alkanethiolates with those of SAMs composed of molecules having different structure (e.g., SAMs of aromatic molecules) requires that $d$ be given in $\AA$.

Statistical Analysis of Data for Current Density Across SAMs. We estimated $J_{o}$ $\left(\mathrm{A} \cdot \mathrm{cm}^{-2}\right)$ and $\beta\left(\AA^{-1} ; \mathrm{nC}^{-1}\right)$ by least-squares linear regression analysis of values of $<\log |J|>$ versus the width $d$ of the tunnel barrier established by the $n$-alkanethiolates, which we assume to be in their all-trans configuration; $<\log |J|>$ is the Gaussian mean value of data for $\log |J|$, where $J$ is the current density (measured in $\mathrm{A} \cdot \mathrm{cm}^{-2}$ at $\mathrm{V}=+0.5 \mathrm{~V}$, and defined for the geometrical contact area estimated by optical microscopy) through SAMs.

Because experimental data for $\log |J|$ are approximately normally distributed for all SAMs, we extracted mean values $(<\log |J|>)$ and standard deviations $\left(\sigma_{\log }\right)$ from Gaussian fits (calculated with standard least-squares fitting ${ }^{18}$ ) to histograms of data.

Estimation of the Specific Resistance of $\mathrm{Ga}_{2} \mathrm{O}_{3} / \mathbf{E G a I n}$ Tips. We estimated the apparent (that is, not corrected for the effective electrical contact area) specific resistance $R_{\mathrm{Ga}_{2} \mathrm{O}_{3}}$ (the resistance per unit area, $\Omega \cdot \mathrm{cm}^{2}$ ) of the $\mathrm{Ga}_{2} \mathrm{O}_{3} / \mathrm{EGaIn}$ conical tips from the specific resistance of junctions with structure $\mathrm{HOPG} / / \mathrm{Ga}_{2} \mathrm{O}_{3} / \mathrm{EGaIn}$ (HOPG = Highly Ordered Pyrolytic Graphite). Because both HOPG and bulk EGaIn are highly conductive materials $\left(R<10^{-6} \Omega \cdot \mathrm{cm}^{2}\right)$, the resistance of these junctions is dominated by the resistance of the $\mathrm{Ga}_{2} \mathrm{O}_{3}$ layer. The advantages of using HOPG surfaces in measurements of contact conductance are that (i) they provide ultra-flat substrates with sub-nanometer roughness; (ii) they do not oxidize in air; and (iii) they can be easily regenerated before each experiment. 


\section{Estimation of the Effective Electrical Contact Area in $\mathrm{Ag}^{\mathrm{TS}}-\mathrm{SAM} / / \mathrm{Ga}_{2} \mathrm{O}_{3} / \mathrm{EGaIn}$ -}

Conical Tip Junctions. We define the ratio of the effective electrical contact area $\left(A_{\text {elec }}\right)$ to the geometrical contact area estimated by optical microscopy $\left(A_{g e o}\right)$ as (Eq. 5):

$$
\frac{A_{\text {elec }}}{A_{\text {geo }}}=\alpha_{\text {tip }} \cdot \alpha_{\text {SAM }}
$$

where $\alpha_{\text {tip }}$ and $\alpha_{\text {SAM }}$ are the fractions of the geometrical surface areas that are available for contact respectively in $\mathrm{Ga}_{2} \mathrm{O}_{3} / \mathrm{EGaIn}$ conical tips and in $\mathrm{Ag}^{\mathrm{TS}}$-SAM substrates; these fractions are defined as the ratios of the effective electrical contact areas to the geometrical contact areas of conical tips $\left(\alpha_{\text {tip }}\right)$, and $\mathrm{Ag}^{\mathrm{TS}}$-SAM $\left(\alpha_{\text {SAM }}\right)$ in contact with an ideally flat electrode (that is, an ideal electrode with a surface topography that does not reduce the effective electrical contact area). We estimated $\alpha_{\text {tip }}$ and $\alpha_{\text {SAM }}$ separately.

\section{Estimation of the fraction of the geometrical surface area of conical tips available}

for contact $\left(\alpha_{t i p}\right)$. We estimated $\alpha_{t i p}$ as the ratio of the current density $J_{t i p}$ flowing through $\mathrm{Ga}_{2} \mathrm{O}_{3} /$ EGaIn-conical tips, to the current density $J_{H g}$ yielded by $\mathrm{Hg}$-drop electrodes, in junctions formed with a standard substrate. We assume that $\mathrm{Hg}$-drop electrodes have smooth, conformal surfaces with an area available for contact close to the geometrical surface area $\left(\alpha_{\mathrm{Hg}} \approx 1\right)$. We used $\mathrm{Fe}^{\mathrm{TS}} / \mathrm{Fe}_{2} \mathrm{O}_{3}\left(\mathrm{Fe}^{\mathrm{TS}}=\right.$ template-stripped iron substrate $)$ as standard substrate because it does not amalgamate with $\mathrm{Hg}$. We grew the $\mathrm{Fe}_{2} \mathrm{O}_{3}$ layer thermally with a specific resistance $\left(R_{\mathrm{Fe}_{2} \mathrm{O}_{3}}\right.$ $\sim 10 \Omega \cdot \mathrm{cm}^{2}$ ) that was $\sim 10^{4}$ higher than the apparent specific resistance of the $\mathrm{Ga}_{2} \mathrm{O}_{3}$ layer estimated using $\mathrm{HOPG}\left(R_{\mathrm{Ga}_{2} \mathrm{O}_{3}}=3.3 \times 10^{-4} \Omega \cdot \mathrm{cm}^{2}\right)$. Because $R_{\mathrm{Ga}_{2} \mathrm{O}_{3}}<<R_{\mathrm{Fe}_{2} \mathrm{O}_{3}}$ by experimental design, the specific resistances of both $\mathrm{Ga}_{2} \mathrm{O}_{3} / \mathrm{EGaIn}$ and $\mathrm{Hg}$-drop based junctions are 
determined by $R_{\mathrm{Fe}_{2} \mathrm{O}_{3}}$, and, at a given voltage $\mathrm{V}$, they must give the same effective current densities (Eq. (6)):

$\frac{I_{\text {tip }}}{A_{\text {tip }}}=\frac{V}{R_{\mathrm{Fe}_{2} \mathrm{O}_{3}}}=\frac{I_{\mathrm{Hg}}}{A_{\mathrm{Hg}}}$

In Eq. $6, I_{\text {tip }}$ is the current flowing through the effective electrical contact area $A_{\text {tip }}$ in junctions using $\mathrm{Ga}_{2} \mathrm{O}_{3} /$ EGaIn conical tips, and $I_{H g}$ is the current through the effective electrical contact area $A_{H g}$ in junctions using Hg-drops.

We define the effective electrical contact area for $\mathrm{Fe}^{\mathrm{TS}} / \mathrm{Fe}_{2} \mathrm{O}_{3} / / \mathrm{Ga}_{2} \mathrm{O}_{3} / \mathrm{EGaIn}$ junctions as $A_{\text {tip }}=A_{\text {geo,tip }} \cdot \alpha_{\text {tip }} \cdot \alpha_{\mathrm{Fe}_{2} \mathrm{O}_{3}}$, where $A_{\text {geo,tip }}$ is the geometrical contact area of $\mathrm{Ga}_{2} \mathrm{O}_{3} /$ EGaIn conical tips on the $\mathrm{Fe}_{2} \mathrm{O}_{3}$ substrate. Analogously, we define the effective electrical contact area of $\mathrm{Fe}^{\mathrm{TS}} / \mathrm{Fe}_{2} \mathrm{O}_{3} / / \mathrm{Ga}_{2} \mathrm{O}_{3} / \mathrm{EGaIn}$ junctions as $A_{\mathrm{Hg}}=A_{g e o, \mathrm{Hg}} \cdot \alpha_{\mathrm{Fe}_{2} \mathrm{O}_{3}}$, with $A_{g e o, \mathrm{Hg}}$ being the geometrical contact area of $\mathrm{Hg}$-drops on the $\mathrm{Fe}_{2} \mathrm{O}_{3}$ substrate. The fraction $\alpha_{\mathrm{Fe}_{2} \mathrm{O}_{3}}$ quantifies the contribution of the roughness of the $\mathrm{Fe}_{2} \mathrm{O}_{3}$ layer to the effective contact area. Equation 6 thus becomes (Eq. 7): $\frac{I_{t i p}}{A_{g e o, t i p} \cdot \alpha_{t i p} \cdot \alpha_{\mathrm{Fe}_{2} \mathrm{O}_{3}}}=\frac{V}{R_{\mathrm{Fe}_{2} \mathrm{O}_{3}}}=\frac{I_{\mathrm{Hg}}}{A_{g e o, \mathrm{Hg}} \cdot \alpha_{\mathrm{Fe}_{2} \mathrm{O}_{3}}}$

The fraction $\alpha_{\mathrm{Fe}_{2} \mathrm{O}_{3}}$ was the same for both junctions because we used the same $\mathrm{Fe}^{\mathrm{TS}} / \mathrm{Fe}_{2} \mathrm{O}_{3}$ chip for all measurements; we thus obtain (Eq. 8):

$\frac{I_{\text {tip }}}{A_{\text {geo }, \text { tip }} \cdot \alpha_{\text {tip }}}=\frac{V}{R_{\mathrm{Fe}_{2} \mathrm{O}_{3}}}=\frac{I_{\mathrm{Hg}}}{A_{\text {geo }, \mathrm{Hg}}}$

Experimental current densities, however, are calculated for the geometrical contact areas, that is (Eq. 9):

$$
J_{\text {tip }}=\frac{I_{\text {tip }}}{A_{\text {geo,tip }}} \text { and } J_{\mathrm{Hg}}=\frac{I_{\mathrm{Hg}}}{A_{g e o, \mathrm{Hg}}}
$$


Here, $J_{\text {tip }}$ is the experimental current density for junctions formed with $\mathrm{Ga}_{2} \mathrm{O}_{3} / \mathrm{EGaIn}$ tips, and $J_{H g}$ is the experimental current density for junctions formed with $\mathrm{Hg}$-drops junctions. By substituting Eq. 9 into Eq. 8 we obtain (Eq. 10):

$$
\alpha_{\text {tip }}=\frac{J_{t i p}}{J_{H g}}
$$

We estimated $J_{t i p}$ and $J_{H g}$ as mean values $<J_{t i p}>$ and $<J_{H g}>$ of data for current density at $\mathrm{V}=+0.5 \mathrm{~V}$ - collected following the "1/3/20" protocol - for $\mathrm{Fe}^{\mathrm{TS}} / \mathrm{Fe}_{2} \mathrm{O}_{3} / /$ $\mathrm{Ga}_{2} \mathrm{O}_{3} /$ EGaIn-conical tips, and for $\mathrm{Fe}^{\mathrm{TS}} / \mathrm{Fe}_{2} \mathrm{O}_{3} / / \mathrm{Hg}$-drop junctions.

\section{Estimation of the fraction of the geometrical surface of $\mathbf{A g}^{\mathrm{TS}}$-SAM substrates}

available for contact $\left(\alpha_{S A M}\right)$. We estimated $\alpha_{S A M}$ from digital analysis of a Scanning Tunneling Microscopy (STM) image of a $\mathrm{Ag}^{\mathrm{TS}}$-SAM substrate using Eq. 11:

$$
\alpha_{S A M}=\frac{q}{Q}
$$

where $q$ is the number of pixels within $2 \AA$ from the top-most average plane of the digital image, and $Q$ is the total number of pixels. The length used $(2 \AA)$ represents the variation of the length of the tunnel gap $\left( \pm 1.0 \AA\right.$, that is, $\left.\sim \pm \sigma_{\log }\right)$ calculated from the average standard deviation of $\log |J|\left(\sigma_{\log }=0.3\right)$ considering $\beta=0.75 \AA^{-1}$ for $n$-alkanethiolates. We used a $900 \mathrm{~nm} \times 900 \mathrm{~nm}$ STM image of a $\mathrm{Ag}^{\mathrm{TS}}-\mathrm{SC}_{10}$ substrate that did not present valleys or asperities that would require, for the estimation of $\alpha_{\text {SAM }}$, that we take into account the partial adaptation of the $\mathrm{Ga}_{2} \mathrm{O}_{3} / E$ GaIn tip to the topography of the surface.

\section{Results and Discussion}

The yield of working junction is independent of the length of the molecule. We observed an average yield of working junction of $\sim 90 \%$ for all chain lengths (including $n=0$ ). For 
the first time, we have been able to measure charge transport through SAMs composed of short molecules of $\mathrm{SC}_{\mathrm{n}}$ (n=0 to 6 ); the yields of working junctions for these short SAMs were approximately the same as those for the longer ones $(n=8$ to $n=18)$. Yields of working junctions for individual $n$-alkanethiolates are summarized in Table S1.

Statistical Analysis of Data for $\log |\boldsymbol{J}|$. Figure 4 summarizes histograms of data for $\log |J|$ (at $\mathrm{V}=+0.5 \mathrm{~V}$ ) for $\mathrm{SAMs}$ of $n$-alkanethiolates from $\mathrm{SC}_{0}$ to $\mathrm{SC}_{18}$ formed on $\mathrm{Ag}^{\mathrm{TS}}$. These histograms approximately fitted Gaussian curves, from which we extracted the mean values $<\log |J|>$ and standard deviations $\sigma_{\log }$. These fits yielded values of $\sigma_{\log } \sim 0.3$ (which corresponds to values of $J(\mathrm{~V})$ from $0.5 \times J(\mathrm{~V})$ to $2 \times J(\mathrm{~V}))$. For all molecules, Gaussian mean, median, and mode (the value of $\log |J|$ with the highest frequency) differed by less than 0.1 . Statistical analysis of histograms for $\log |J|$ at $\mathrm{V}=-0.5 \mathrm{~V}$ (summarized in Figure S3) led to mean values $<\log |J|>$ and standard deviation $\sigma_{\log }$ similar to those obtained for $\mathrm{V}=+0.5 \mathrm{~V}$, revealing a symmetry of tunneling rates in the range of voltages $\mathrm{V}= \pm 0.5 \mathrm{~V}$.

Calculating $\boldsymbol{J}_{\boldsymbol{o}}$ and $\boldsymbol{\beta}$. Figure 1 shows that values of $<\log |J|>$ (both at $\mathrm{V}=+0.5 \mathrm{~V}$, and $\mathrm{V}=-0.5 \mathrm{~V})$ decreased linearly with the length $d$ of the $\mathrm{S}-\left(\mathrm{CH}_{2}\right)_{\mathrm{n}}-\mathrm{H}$ group ( $d$ is also given as the number of carbon atoms $\mathrm{nC}$ on the top-axis of the plot). This plot is compatible with the simplified Simmons equation for tunneling (Equation 1). For $\mathrm{V}=+0.5 \mathrm{~V}$, the linear regression analysis yielded $<\log \left|J_{o}\right|>=3.6 \pm 0.3\left(\left[J_{o}\right]=\mathrm{A} \cdot \mathrm{cm}^{-2}\right)$; this value was statistically indistinguishable from that obtained for $\mathrm{V}=-0.5 \mathrm{~V}\left(<\log \left|J_{o}\right|>=3.4 \pm 0.3,\left[J_{o}\right]=\mathrm{A} \cdot \mathrm{cm}^{-2}\right)$. These values of $J_{o}$ estimate the current density injected across the $\mathrm{Ag}^{\mathrm{TS}}-\mathrm{S}$ and the $\mathrm{Ga}_{2} \mathrm{O}_{3} / / \mathrm{SAM}$ interfaces into the alkyl chain.

For both $\mathrm{V}=+0.5 \mathrm{~V}$ and $\mathrm{V}=-0.5 \mathrm{~V}$, the slope of the linear regression analysis gave a value of the tunnel decay factor of $\beta=0.75 \pm 0.02 \AA^{-1}\left(\beta=0.92 \pm 0.02 \mathrm{nC}^{-1}\right)$. 
Ohmic Electrical Transport Through the $\mathbf{G}_{\mathbf{2}} \mathbf{O}_{3}$ layer. The HOPG// $\mathrm{Ga}_{2} \mathrm{O}_{3} / \mathrm{EGaIn}$ junction yielded $100 \%$ of working junctions. For this junction, $J$ varied linearly with the voltage (Figure 5) and confirmed the ohmic transport across the $\mathrm{Ga}_{2} \mathrm{O}_{3}$ layer previously reported ${ }^{34}$. Linear regression of the $J-\mathrm{V}$ average trace yielded an ohmic conductivity (slope) of $\sim 3.0 \times 10^{3} \mathrm{~A} \cdot \mathrm{cm}^{-2} \cdot \mathrm{V}^{-1}$, from which we estimated an apparent specific resistance (i.e., the resistance per unit area, and not corrected for the effective electrical contact area) of the $\mathrm{Ga}_{2} \mathrm{O}_{3}$ layer of $R_{\mathrm{Ga}_{2} \mathrm{O}_{3}}=3.3 \times 10^{-4} \Omega \cdot \mathrm{cm}^{2}$. The measured specific resistance of the $\mathrm{Ga}_{2} \mathrm{O}_{3}$ layer was lower - by a factor of $\sim 10$ - than the specific resistance of the SAM of the shortest alkanethiolate $\mathrm{SC}_{1}\left(R_{S C_{1}}=1 \times 10^{-3} \Omega \cdot \mathrm{cm}^{2}\right)$.

In our previous work ${ }^{34}$, we used copper wires with a diameter of $80 \mu \mathrm{m}$ to estimate a resistance of the $\mathrm{Ga}_{2} \mathrm{O}_{3}$ layer of $R_{\mathrm{Ga}_{2} \mathrm{O}_{3}}=4 \times 10^{-2} \Omega \cdot \mathrm{cm}^{2}$; we believe that this estimation is less accurate than the new one we report here for two reasons: i) due to the shape and size of the wires, we were not able to measure accurately the geometrical contact area of the $\mathrm{Cu}$-wires on $\mathrm{Ga}_{2} \mathrm{O}_{3} /$ EGaIn droplets; and ii) we did not take into account the contribution of the native oxide $\mathrm{CuO}_{\mathrm{x}}$ passivating the surface of the $\mathrm{Cu}$ wires to the resistance of the $\mathrm{Cu} / \mathrm{CuO}_{\mathrm{x}} / / \mathrm{Ga}_{2} \mathrm{O}_{3} / \mathrm{EGaIn}$ contact. These $\mathrm{Cu} / \mathrm{CuO}_{\mathrm{x}} / / \mathrm{Ga}_{2} \mathrm{O}_{3} / \mathrm{EGaIn}$ contacts, however, exhibited ohmic transport.

The effective electrical contact area of $\mathrm{Ag}^{\mathrm{TS}}$-SAM//Ga $\mathrm{O}_{3} / \mathrm{EGaIn}$ junctions using flattened conical tips was $\sim \mathbf{1 0}^{-4}$ the geometrical contact area. To estimate the effective electrical contact area, we calculated the fraction of the surface area available for contact for $\mathrm{Ga}_{2} \mathrm{O}_{3} /$ EGaIn conical tips $\left(\alpha_{\text {tip }}\right)$, and for $\mathrm{Ag}^{\mathrm{TS}}$-SAM substrates $\left(\alpha_{\text {SAM }}\right)$.

Estimation of $\alpha_{\text {tip }}$. Figure 6 shows the histograms for data for $\log |J|$ and the relative Gaussian fits for junctions having structure, A) $\mathrm{Fe}^{\mathrm{TS}} / \mathrm{Fe}_{2} \mathrm{O}_{3} / \mathrm{Hg}$-drops, and $\mathrm{B}$ ) 
$\mathrm{Fe}^{\mathrm{TS}} / \mathrm{Fe}_{2} \mathrm{O}_{3} / / \mathrm{Ga}_{2} \mathrm{O}_{3} / \mathrm{EGaIn}$ (flattened conical tips). The resistance of the junction formed with $\mathrm{Hg}$-drops was determined by the specific resistance of the $\mathrm{Fe}_{2} \mathrm{O}_{3}$ layer. Because, by experimental design, the specific resistance of this $\mathrm{Fe}_{2} \mathrm{O}_{3}$ layer was $\sim 10^{4}$ the specific resistance at of the $\mathrm{Ga}_{2} \mathrm{O}_{3}$ layer (estimated from measurements of contact resistance of $\mathrm{Ga}_{2} \mathrm{O}_{3} / \mathrm{EGaIn}$ tips on HOPG), also the specific resistance of the $\mathrm{Ga}_{2} \mathrm{O}_{3} / \mathrm{EGaIn}$ based junctions was determined by the resistance of the $\mathrm{F}_{2} \mathrm{O}_{3}$ layer. Having approximately the same specific resistance $\left(\sim R_{\mathrm{Fe}_{2} \mathrm{O}_{3}}\right)$, at a fixed voltage, both $\mathrm{Ga}_{2} \mathrm{O}_{3} / \mathrm{EGaIn}$ conical tips and $\mathrm{Hg}$-drops should have given the same value of current density. The average value (at $\mathrm{V}=+0.5 \mathrm{~V},<\log \left|J_{\text {tip }}\right|>=-4.2 \pm 0.4$ ) of the current density measured with $\mathrm{Ga}_{2} \mathrm{O}_{3} /$ EGaIn conical tips, however, was approximately $10^{-3}$ the average current density measured with the Hg-drop (at $\mathrm{V}=+0.5 \mathrm{~V},<\log \left|J_{H g}\right|>=-1.3 \pm 0.1$ ). Using these experimental values of current densities, from Eq. 10 we estimated $\alpha_{\text {tip }}=10^{-3.0 \pm 0.5}$. We emphasize that, by experimental design, the difference in $J(\mathrm{~V})$ between $\mathrm{Ga}_{2} \mathrm{O}_{3} / \mathrm{EGaIn}$ and Hg-drop-based junctions cannot be explained by the difference between the resistances of $\mathrm{Hg}$ and of the $\mathrm{Ga}_{2} \mathrm{O}_{3}$ layer.

Estimation of $\alpha_{\text {SAM }}$. Figure 7-A shows a STM image of a SAM of $\mathrm{SC}_{10}$ on $\mathrm{Ag}^{\mathrm{TS}}$. By digital analysis of the pixels (Eq. 11), we estimated that $\sim 12 \%$ of the geometrical surface area of the $\mathrm{Ag}^{\mathrm{TS}}$-SAM substrate was available for contact (Figure 7-B), that is, $\alpha_{S A M} \sim 10^{-1}$. (We reported the STM surface profiles of two different regions of the sample in Figure S4).

Correction for effective contact area yields $\boldsymbol{J}_{\boldsymbol{o}} \sim \mathbf{1 0}^{\mathbf{7 . 6 \pm 0 . 8}} \mathbf{A} \cdot \mathbf{c m}^{-2}$. Using, in Eq. 5, values obtained for the fractions of the surface available for contact respectively for $\mathrm{Ga}_{2} \mathrm{O}_{3} / \mathrm{EGaIn}$ conical tips $\left(\alpha_{\text {tip }}=10^{-3.0 \pm 0.5}\right)$, and for $\operatorname{Ag}^{\mathrm{TS}}$-SAM substrates $\left(\alpha_{\text {SAM }}=10^{-1}\right)$, for junctions having structure $\mathrm{Ag}^{\mathrm{TS}}$-SAM// $\mathrm{Ga}_{2} \mathrm{O}_{3} / \mathrm{EGaIn}$ using flattened conical tips, we estimated that the effective electrical contact area is approximately $A_{\text {elec }}=10^{-4.0 \pm 0.5} \cdot A_{\text {geo }}$. The correction of the experimental 
value of $<\log \left|J_{o}\right|>$ (Figure 1) for the effective electrical contact area provided an estimate for the injection current density of $\left\langle\log \left|J_{o}\right|>=7.6 \pm 0.8\left(J_{o} / \mathrm{A} \cdot \mathrm{cm}^{-2}\right)\right.$. This corrected value of $J_{o}$ was compatible with those estimated for single-molecules approaches ${ }^{5-9}$, and with those reported for large-area junctions using graphene ${ }^{11}$ and evaporated- $\mathrm{Au}^{12}$ top-electrodes used for SAMs formed on flat substrates (Figure 8; we marked as " $\mathrm{Ga}_{2} \mathrm{O}_{3} / \mathrm{EGaIn}$ corrected (estimate)" the value of $<\log \left|J_{o}\right|>$ corrected for the estimated value of the effective electrical contact area).

\section{Conclusion}

$\mathrm{Ga}_{2} \mathrm{O}_{3} / \mathbf{E G a I n - b a s e d ~ j u n c t i o n s ~ s h o w ~ a ~ r e p r o d u c i b l e ~ v a l u e ~ o f ~ t h e ~ e f f e c t i v e ~ e l e c t r i c a l ~}$

contact area. In this paper we have described a simple technique for the fabrication of what we call "flattened" $\mathrm{Ga}_{2} \mathrm{O}_{3} /$ EGaIn conical tips. Using these tips reduced the variability in values of $\log |J|$ from $\sigma_{\log }=0.5-1.1$ for as-fabricated tips to $\sigma_{\log } \sim 0.3$. The procedure used to flatten the conical tips also retained the convenience of using conical EGaIn top-electrodes.

The comparison of the electrical behavior of smooth Hg-drop top-electrodes with that of flattened (but intrinsically rough) $\mathrm{Ga}_{2} \mathrm{O}_{3} / E$ GaIn conical tips suggests that in junctions having structure $\mathrm{Ag}^{\mathrm{TS}}$-SAM$/ / \mathrm{Ga}_{2} \mathrm{O}_{3} / \mathrm{EGaIn}$ the effective electrical contact area is $\mathrm{A}_{\text {elect }}=10^{-4.0 \pm 0.5} \cdot \mathrm{A}_{\text {geo }}$, where $\mathrm{A}_{\text {geo }}$ is the geometrical contact area measured by optical microscopy. This value of $A_{\text {elec }}-$ a value that is small, but compatible with estimates of the area of physical contact from measurements of adhesion and friction between surfaces ${ }^{21-27}$ — was reproducible from junction to junction. The high reproducibility may perhaps surprise, but it is plausibly attributable to the low compressibility of the SAM, and to the (roughly) constant load applied to the contact. Once a stable contact is formed on top of the SAM (using the observable wrinkling of the tip apex as an intrinsic sensor of the load applied to the junctions), the load applied to the contact cannot be 
significantly further varied: pushing the $\mathrm{Ga}_{2} \mathrm{O}_{3} /$ EGaIn conical tip against the substrate simply deforms the tip (by making it bulge laterally), and increases (perhaps slightly) the geometrical contact area. We can also infer that following the same procedure for the fabrication of different tips gives surfaces of the $\mathrm{Ga}_{2} \mathrm{O}_{3}$ layer with a reproducible roughness. We believe that the reproducibility of the roughness of the $\mathrm{Ga}_{2} \mathrm{O}_{3}$ layer and the invariance of the load applied to the $\mathrm{SAM} / / \mathrm{Ga}_{2} \mathrm{O}_{3} / \mathrm{EGaIn}$ contacts result in a reproducible values of $\mathrm{A}_{\text {eled }} / \mathrm{A}_{\text {geo. }}$.

For $\mathrm{Ga}_{2} \mathrm{O}_{3} / \mathrm{EGaIn}$ top-electrodes, the resistance of the oxide layer is not important. For junctions formed with flattened $\mathrm{Ga}_{2} \mathrm{O}_{3} / \mathrm{EGaIn}$ conical tips, we observed a linear variation of values of $<\log |J|>$ with a constant slope $\left(\beta=0.75 \pm 0.02 \AA^{-1} ; \beta=0.92 \pm 0.02 \mathrm{nC}^{-1}\right)$ for tunneling through SAMs of $\mathrm{SC}_{\mathrm{n}}$ in the range of lengths from $\mathrm{n}=0$ to $\mathrm{n}=18$. We would not observe this linearity over the complete range (values of current densities varied over seven orders of magnitude) if the resistance of the $\mathrm{Ga}_{2} \mathrm{O}_{3}$ layer were contributing to the resistance of the junction.

We do not know, however, if this value of $\beta$ estimated from measurements of tunneling across $\mathrm{Ag}^{\mathrm{TS}}-\mathrm{SC}_{\mathrm{n}} / / \mathrm{Ga}_{2} \mathrm{O}_{3} / \mathrm{EGaIn}$ junctions is the true value of $\beta$ characteristic for tunneling across $n$-alkanethiolates. Values of $\beta$ for tunneling through $n$-alkanethiolates vary by $\sim 20 \%$ across different methods (Figure 8); whether this range is due to true variations of the characteristics of SAMs of $n$-alkanethiolates (e.g., the orientation, order, and density of the packing of the chains, differences in the topography of the supporting metal electrode), or to still-unresolved artifacts characteristic of the different junctions, remains unresolved.

The consensus value of $J_{o}$ for tunneling through $n$-alkanethiolates lies in the range $10^{6}-10^{8} \mathbf{A} \cdot \mathbf{c m}^{-2}$ (at $\mathbf{V}=+\mathbf{0 . 5} \mathrm{V}$ ). For junctions with structure $\mathrm{Ag}^{\mathrm{TS}}-\mathrm{SC}_{\mathrm{n}} / / \mathrm{Ga}_{2} \mathrm{O}_{3} / \mathrm{EGaIn}$ (conical tips), converting the value of the geometrical contact area $\left(\mathrm{A}_{\text {geo }}\right)$ into the estimated value of the 
effective electric contact area $\left(\mathrm{A}_{\text {elec }}=10^{-4.0 \pm 0.5} \cdot \mathrm{A}_{\mathrm{geo}}\right)$ led to a value of the effective injection current density $J_{o}=10^{7.6 \pm 0.8} \mathrm{~A} \cdot \mathrm{cm}^{-2}$. This corrected value of $J_{o}$ is compatible with those obtained for junctions using smooth and flat electrodes, that is, junctions using electrodes that maximize the value of $A_{\text {eled }} / A_{\text {geo }}$. Junctions formed with Hg-drops on top of alkyl chains anchored to silicon surfaces $^{3,4}$ yielded $J_{o} \sim 10^{6}-10^{6.5} \mathrm{~A} \cdot \mathrm{cm}^{-2}$, whereas large-area junctions using flat metal substrates (RMS 0.7 $\AA$ ) and flat top-electrodes (e.g.; graphene ${ }^{11}$ ), or compliant, evaporated $\mathrm{Au}$ electrodes ${ }^{12}$, yielded rates of charge transport of $J_{o} \sim 10^{8} \mathrm{~A} \cdot \mathrm{cm}^{-2}$. These results consolidate a consensus value of the injection current for SAMs of $n$-alkanethiolates in the range $10^{6}-10^{8}$ $\mathrm{A} \cdot \mathrm{cm}^{-2}(\mathrm{~V}=+0.5 \mathrm{~V})$ (Figure 8$)$

Values of $J_{o}<10^{6} \mathrm{~A} \cdot \mathrm{cm}^{-2}$ (Figure 8, gray field) might imply an overestimation of the effective electrical contact area. In junctions using rough $\mathrm{Ga}_{2} \mathrm{O}_{3} / \mathrm{EGaIn}$ top-electrodes, or rough, as-evaporated metal substrates (and using $\mathrm{Hg} /$ polymer $^{13}$, or $\mathrm{Hg}_{-} \mathrm{SAM}^{15}$ top-electrodes), the assumption that $\mathrm{A}_{\text {elec }}$ equals $\mathrm{A}_{\text {geo }}$ leads to values of $J_{o}$ in the range $10^{2}-10^{3} \mathrm{~A} \cdot \mathrm{cm}^{-2}$. These low experimental values of $J_{o}$ cannot be rationalized by an hypothetical high resistance of the topelectrodes: $\mathrm{Hg}$ /polymer and $\mathrm{Ga}_{2} \mathrm{O}_{3} / \mathrm{EGaIn}$ are highly conductive materials. We believe that, due to the roughness of the electrodes, the value of $\mathrm{A}_{\mathrm{geo}}$ overestimates the effective electrical area of contact $\left(\mathrm{A}_{\text {elec}}\right)$ of these junctions, thus resulting in an underestimation of $J_{o}$.

The value $10^{9} \mathrm{~A} \cdot \mathrm{cm}^{-2}$ might be a hypothetical upper limit to possible experimental estimations of $J_{o}$ for SAMs of $n$-alkanethiolates. We estimated this value of $J_{o}$ by extrapolating the value of $J_{o}\left(\sim 1 \times 10^{-5} \mathrm{~A} \cdot \mathrm{mol}^{-1}\right)$ measured for single $n$-alkanethiolates in STM-based junctions ${ }^{5-7,10}$ (Figure 8 ) to the value of $J_{o}$ for the theoretical number of molecules per $\mathrm{cm}^{2}$ of the SAM (on $\mathrm{Au}\left(111\right.$ ), this number is $\sim 4.5 \times 10^{14} \mathrm{~mol} \cdot \mathrm{cm}^{-2}$ ). This extrapolation assumes that the SAM is free of defects and has an homogeneous density of molecules, and that all the molecules 
of this ideal SAM are in contact with the top-electrode; these assumptions are unrealistic in experiments with large-area junctions, for which values of $J_{o}$ in the range $10^{6}-10^{8} \mathrm{~A} \cdot \mathrm{cm}^{-2}$ are more probable (Figure 8). The values of $\mathrm{A}_{\text {elec }} / \mathrm{A}_{\text {geo }}$ for these different techniques, however, are unknown, and comparing values of $J_{o}(\mathrm{~V})$ will require the use of a common standard to take into account differences in measured values of $J(\mathrm{~V})$. We recommend using $\mathrm{SAMs}$ of $\mathrm{SC}_{10}$ and $\mathrm{SC}_{16}$ as standards for the calibration of values of $J(\mathrm{~V})$.

\section{The absolute value of the effective electrical contact area is not important in} physical-organic studies; its reproducibility is important. Using $\mathrm{Ga}_{2} \mathrm{O}_{3} / \mathrm{EGaIn}$ flattened conical tips as top-electrodes yielded a (roughly) constant value of the standard deviation $\left(\sigma_{\log } \sim 0.3\right)$ of value of $\log |J|$ across SAMs of individual $n$-alkanehthiolates, and a value of the tunneling decay factor $\beta=0.75 \pm 0.02 \AA^{-1}$ ( or $\beta=0.92 \pm 0.02 \mathrm{nC}^{-1}$ ) that falls in the $90 \%$ consensus range of values of $\beta$ estimated by different techniques (Figure 8). These results indicate that, in $\mathrm{Ag}^{\mathrm{TS}}$-SAM $/ / \mathrm{Ga}_{2} \mathrm{O}_{3} / \mathrm{EGaIn}$ junctions, the value of $\mathrm{A}_{\text {elec }} / \mathrm{A}_{\text {geo }}$ is (surprisingly) reproducible from junction to junction, and that variations of measured values of tunneling currents across different SAMs are primarily due to differences in the lengths of the $n$-alkanethiolates. The dispersion in data collected with flattened $\mathrm{Ga}_{2} \mathrm{O}_{3} / \mathrm{EGaIn}$ conical tips was smaller than that observed for asfabricated conical tips; flattening of the tips, however, had no effects on the mean values (i.e., $<\log |J|>)$ of the distributions of experimental values of $\log |J|$.

\section{$\mathrm{Ga}_{2} \mathrm{O}_{3} / \mathrm{EGaIn}$ top-electrodes are a convenient methodology for physical-organic}

studies of charge tunneling. Table 2 summarizes advantages and disadvantages that we identified for the most common techniques used for studying charge transport through molecules anchored to conductive substrates. From this comparison, $\mathrm{Ga}_{2} \mathrm{O}_{3} / \mathrm{EGaIn}$ top-electrodes emerge as a particularly convenient technology for physical-organic studies of charge transport through 
molecules. Physical-organic approaches, which examine only trends in $J(V)$ as a function of the structure of the molecules making up the SAM, require that the properties of the top-and bottom-interfaces of these different types of junction are unchanged across a series of measurements. The roughness of the $\mathrm{Ga}_{2} \mathrm{O}_{3}$ layer gives an uncertain morphology to the $\mathrm{SAM} / / \mathrm{Ga}_{2} \mathrm{O}_{3}$ interface, but, so long as the properties of this interface remain constant, physicalorganic protocols can be applied to the study of charge transport through SAMs, even though the $\mathrm{SAM} / / \mathrm{Ga}_{2} \mathrm{O}_{3}$ interface is not well defined (None of the interfaces of the methods listed in Table 2 is, however, entirely defined).

The simplicity and rapidity in assembling junctions using $\mathrm{Ga}_{2} \mathrm{O}_{3} / \mathrm{EGaIn}$ top-electrodes that make it possible to collect large numbers of data $\left(\sim 10^{3}\right.$ per day) are currently unmatched by any other method listed in Table 2 . We stress that collection of large numbers of data is critical to validating the statistical significance of studies of structure-tunneling relation by physicalorganic protocols.

$\mathrm{Ga}_{2} \mathrm{O}_{3} / \mathbf{E G a I n}$ tips do not damage the SAM. $\mathrm{Ga}_{2} \mathrm{O}_{3} / \mathrm{EGaIn}$ top-electrodes enable a rapid collection of data because they do not require a fine control of the load to form non-damaging contacts on top of the SAM. The load applied to contacts using soft $\mathrm{Ga}_{2} \mathrm{O}_{3} / \mathrm{EGaIn}$ conical tipsor liquid metal electrodes in general—cannot be significantly varied; pushing the $\mathrm{Ga}_{2} \mathrm{O}_{3} / \mathrm{EGaIn}$ electrode against the sample simply increases the geometrical contact area of the junction. By contrast, small variations of the load applied to junctions using solid top-electrodes might change the effective electrical contact area, deform the SAM, and even damage the SAM. With only the exception of Hg-based junctions, all other methods listed in Table 2 use solid top-electrodes; the loads applied to these contacts, however, have never been reported. 


\section{$\mathrm{Ga}_{2} \mathrm{O}_{3} / \mathrm{EGaIn}$ is an affordable technology that might allow comparison of results}

obtained in different laboratories. Several years of work in our laboratory have proved that EGaIn-based junctions are a practical and convenient methodology for physical-organic studies of charge tunneling through SAMs.

So far, the effect of the resistance of the $\mathrm{Ga}_{2} \mathrm{O}_{3}$ layer on the electrical behavior of the junctions, and the value of the effective electrical contact area between SAMs and $\mathrm{Ga}_{2} \mathrm{O}_{3} / \mathrm{EGaIn}$ conical tips have been two major unresolved issues in the interpretation of data obtained with EGaIn-based junctions. In this paper we have shown that the resistance of the $\mathrm{Ga}_{2} \mathrm{O}_{3}$ layer is not important, and that the effective electrical contact area-which is $\sim 10^{-4}$ the geometrical contact area - is reproducible across different experiments; the correction of current densities for this value of the effective electrical contact area led to results for tunneling across $n$-alkanethiolates compatible with those reported for techniques far more complex and expensive than $\mathrm{Ga}_{2} \mathrm{O}_{3} /$ EGaIn electrodes.

Using $\mathrm{Ga}_{2} \mathrm{O}_{3} / \mathrm{EGaIn}$ top-electrodes still requires attention to experimental details (especially the fabrication of the conical tips, and the formation of stable contacts on top of the SAM), but the procedure requires only a few days to learn. The simple (and inexpensive) fabrication of EGaIn-based junctions can be replicated in any laboratory, and the availability of very well-defined references data (such those of Figure 1) for comparison, calibration, and use as standards makes comparisons among data generated in different laboratories straightforward.

\section{Acknowledgement}

This work was supported by a subcontract from Northwestern University from the Department of Energy (DE-SC0000989) for work on synthesis of materials and measurements of charge transport. 
Table 1. Comparison of values of injection tunnel current $J_{o}(\mathrm{~V}=+0.5 \mathrm{~V})$ and tunnel decay

\section{factor $\beta$ for $n$-alkanethiolates $\left(\mathrm{SC}_{\mathrm{n}}\right)$ reported (or estimated) for different types of junctions.}

\begin{tabular}{|c|c|c|c|c|c|c|c|}
\hline & Type of junction & Top-electrode & $\begin{array}{r}\text { Bottom- } \\
\text { electrode }\end{array}$ & Values of $\mathbf{n}$ & $\begin{array}{c}\log \left|J_{0}\right| \\
\mathbf{J}_{0} / \mathbf{A c m}^{-2} \\
\end{array}$ & $\begin{array}{c}\beta \\
\mathbf{n C}^{-1}\end{array}$ & ref \\
\hline 1 & $\mathrm{Au}-\mathrm{SAM} / /$ polymer $/ \mathrm{Hg}^{\mathrm{a}}$ & conductive polymer & AS-DEP & $8,10,12,14,16$ & $\sim 2$ & $1.10 \pm 0.04$ & [13] \\
\hline 2 & $\mathrm{Ag}-\mathrm{SAM} / / \mathrm{Ga}_{2} \mathrm{O}_{3} / \mathrm{EGaIn}$ & $\mathrm{Ga}_{2} \mathrm{O}_{3} / \mathrm{EGaIn}$ (cone) & $\mathrm{TS}^{f}$ & $9,11,13,15,17$ & 1.9 & $1.00 \pm 0.02$ & [18] \\
\hline 3 & $\mathrm{Ag}-\mathrm{SAM} / / \mathrm{Ga}_{2} \mathrm{O}_{3} / \mathrm{EGaIn}$ & $\mathrm{Ga}_{2} \mathrm{O}_{3} / \mathrm{EGaIn}$ (cross-bar) & TS & $12,14,16,18$ & $\sim 2.5$ & $0.95 \pm 0.2$ & {$[20]$} \\
\hline 4 & $\mathrm{Ag}-\mathrm{SAM} / / \mathrm{Ga}_{2} \mathrm{O}_{3} / \mathrm{EGaIn}$ & $\mathrm{Ga}_{2} \mathrm{O}_{3} / \mathrm{EGaIn}$ (cone) & TS & $10,12,14,16,18$ & 2.5 & $0.97 \pm 0.02$ & {$[18]$} \\
\hline 5 & $\mathrm{Ag}-\mathrm{SAM} / / \mathrm{Ga}_{2} \mathrm{O}_{3} / \mathrm{EGaIn}$ & $\mathrm{Ga}_{2} \mathrm{O}_{3} / \mathrm{EGaIn}$ (cone) & TS & $10,12,14,16$ & $\sim 3$ & $1.1 \pm 0.2$ & [19] \\
\hline 6 & $\mathrm{Ag}-\mathrm{SAM} / / \mathrm{Ga}_{2} \mathrm{O}_{3} / \mathrm{EGaIn}$ & $\mathrm{Ga}_{2} \mathrm{O}_{3} / \mathrm{EGaIn}$ (cone) & TS & $0,1,2,3,4-18$ & $3.6 \pm 0.3$ & $0.92 \pm 0.02$ & this \\
\hline 7 & $\mathrm{Ag}-\mathrm{SAM} / / \mathrm{SAM}-\mathrm{Hg}^{b}$ & $\mathrm{Hg}-\mathrm{SC}_{16}$ & AS-DEP & $8,10,12,14,16$ & $\sim 3$ & 1.0 & {$[15]$} \\
\hline 8 & $\mathrm{Au}-\mathrm{SAM}-\mathrm{Au}$ & Au Nanoskiving & Nanoskiving & $12,14,16$ & 3.2 & 0.94 & {$[51]$} \\
\hline 9 & $\mathrm{Au}-\mathrm{SAM} / / \mathrm{PEDOT} / \mathrm{Au}$ & PEDOT:PSS & AS-DEP & $8,10,12,14$ & $\sim 5$ & 0.76 & [47] \\
\hline 10 & $\mathrm{Hg}-\mathrm{SAM} / / \mathrm{SAM}-\mathrm{Hg}^{c}$ & $\mathrm{Hg}$ & liquid metal & $18,20,22,24,28$ & $\sim 6$ & 0.9 & {$[50]$} \\
\hline 11 & $\mathrm{Hg}-\mathrm{SAM} / / \mathrm{Hg}^{d}$ & $\mathrm{Hg}$ & liquid metal & $9,10,11,12,15,16,18$ & $\sim 6$ & $1.04 \pm 0.07$ & [49] \\
\hline 12 & nSi-SAM//Hg & $\mathrm{Hg}$ & $\mathrm{n}-\mathrm{Si}^{g}$ & $14,16,18$ & $6.5^{h}$ & $0.9 \pm 0.2$ & {$[3,4]$} \\
\hline 13 & $\mathrm{Au}-\mathrm{SAM} / /$ graphene & graphene & TS & $8,12,16$ & 8.3 & $1.02 \pm 0.1$ & {$[11]$} \\
\hline 14 & $\mathrm{Au}-\mathrm{SAM}-\mathrm{Au}$ & Au evaporated & TS & $8,12,16$ & $\sim 8$ & $0.98 \pm 0.03$ & [12] \\
\hline 15 & $\mathrm{Au}-\mathrm{SAM}-\mathrm{Au}$ & STM tip & Single Cryst ${ }^{i}$ & $6,8,10$ & 8.9 & 1.09 & {$[5-7]$} \\
\hline 16 & $\mathrm{Au}-\mathrm{SAM}-\mathrm{Au}$ & AFM tip & Single Cryst & $6,8,10,12$ & 8.2 & 0.8 & {$[8,9]$} \\
\hline
\end{tabular}

${ }^{a}$ Conductive polymer=polyphenylenevinylene-type polymer; ${ }^{b} J_{o}$ extrapolated at the length of the top-SAM. Including the top-SAM in the tunnel barrier of the junction yields $\log \left|J_{O}\right|=\sim 6 ;{ }^{C} J_{o}$ not reported by the authors of the original paper; we estimated $J_{o}$ from published data; ${ }^{d}$ Junction held under electrochemical control; ${ }^{e}$ AS-DEP: As deposited, rough metal substrate; ${ }^{f}$ TS: Templatestripped, flat metal substrate; ${ }^{g}$ highly n-doped Silicon; ${ }^{h} J_{o}$ calculated from value of contact conductance of a single molecule reported in the original article; ${ }^{I}$ Single Cryst: flat terraces of single crystal surfaces. 
Table 2. Summary of advantages and disadvantages of the most common types of molecular junctions.

\begin{tabular}{|c|c|c|}
\hline Top-electrode & Advantages & Disadvantages \\
\hline $\mathrm{Ga}_{2} \mathrm{O}_{3} / \mathrm{EGaIn}$ & $\begin{array}{l}\text {-Rapid data collection; } \\
\text {-High yield }(90 \%) \text { of working junctions; } \\
\text {-Simple design; } \\
\text {-No damaging contact; } \\
\text {-Conformal contact; } \\
\text {-Insensitive to contact load. } \\
\text {-*For flattened tips, small dispersion in } \\
\quad \text { data for log|J.| }\end{array}$ & $\begin{array}{l}\text {-Surface roughness; } \\
\text {-Limited range of temperature; } \\
\text {-Limited range of voltages; } \\
\text {-Subject to adventitious } \\
\text { contaminations; }\end{array}$ \\
\hline Hg-drops & $\begin{array}{l}\text {-Well defined surface; } \\
\text {-Rapid data collection; } \\
\text {-Conformal contact; } \\
\text {-Non damaging contact; } \\
\text {-Insensitive to contact load. }\end{array}$ & $\begin{array}{l}\text {-Toxic; } \\
\text {-Volatile; } \\
\text {-Amalgamates with Au and Ag; } \\
\text {-Needs hexadecane bath; } \\
\text {-Surface contamination (high surface } \\
\text { energy) }\end{array}$ \\
\hline Hg-SAM & $\begin{array}{l}\text {-Rapid data collection; } \\
\text {-Conformal contact; } \\
\text {-Non damaging contact; } \\
\text {-Insensitive to contact load. }\end{array}$ & $\begin{array}{l}\text {-Toxic; } \\
\text {-Amalgamates with Au and Ag; } \\
\text {-Needs a hexadecane bath; } \\
\text {-Intercalation of molecules from the } \\
\text { top- SAM into the bottom-SAM might } \\
\text { occur; } \\
\text {-Possible lateral movement, or exchange of } \\
\text { SAM on Hg. }\end{array}$ \\
\hline Graphene & $\begin{array}{l}\text {-Well defined top-interface; } \\
\text {-High stability; } \\
\text {-High durability; } \\
\text {-High yield. } \\
\text {-Resistant to a broad range of } \\
\text { temperatures } \\
\text {-Usable at high voltages. }\end{array}$ & $\begin{array}{l}\text {-Complicated experimental protocol; } \\
\text {-Sensitive to load applied to contact; } \\
\text {-Time consuming. }\end{array}$ \\
\hline $\begin{array}{l}\text { Single Molecules } \\
\text { (STM, AFM) }\end{array}$ & $\begin{array}{l}\text {-Rapid collection of data; } \\
\text {-Resistant to a broad range of } \\
\text { temperatures; } \\
\text {-Usable at high voltages. }\end{array}$ & $\begin{array}{l}\text {-Unknown number of contacted } \\
\text { molecules; } \\
\text {-Complicated experimental protocol; } \\
\text {-Time consuming; } \\
\text {-High equipment cost; } \\
\text {-Sensitive to contact load. }\end{array}$ \\
\hline $\begin{array}{l}\text { Conductive } \\
\text { Polymers }\end{array}$ & -Durability. & $\begin{array}{l}\text {-Requires thermal annealing that can } \\
\text { damage the SAM } \\
\text {-Complicated design; } \\
\text { - Possible intercalation of molecules into } \\
\text { the SAM. } \\
\text {-Sensitive to load; } \\
\text {-Time consuming. }\end{array}$ \\
\hline Evaporated Au & -Conformal contact & $\begin{array}{l}\text {-Damaging contact; } \\
\text {-Formation of filaments; } \\
\text {-Difficult experimental protocol; } \\
\text {-Time consuming. }\end{array}$ \\
\hline
\end{tabular}




\section{References}

(1) Simmons, J. G. J. Appl. Phys. 1963, 34, 1793.

(2) Simmons, J. G. J. Appl. Phys. 1963, 34, 2581.

2010, 22, 140 .

(3) Vilan, A.; Yaffe, O.; Biller, A.; Salomon, A.; Kahn, A.; Cahen, D. Adv. Mat.

(4) Yaffe, O.; Scheres, L.; Segev, L.; Biller, A.; Ron, I.; Salomon, A.; Giesbers, M.; Kahn, A.; Kronik, L.; Zuilhof, H.; Vilan, A.; Cahen, D. J. Phys. Chem. C 2010, 114, 10270.

(5) Xu, B. Q.; Tao, N. J. J. Science 2003, 301, 1221.

(6) Chen, F.; Li, X. L.; Hihath, J.; Huang, Z. F.; Tao, N. J. J. Am. Chem. Soc. 2006, $128,15874$.

(7) Suzuki, M.; Fujii, S.; Fujihira, M. Jpn. J. Appl. Phys., Part 1 2006, 45, 2041.

(8) Engelkes, V. B.; Beebe, J. M.; Frisbie, C. D. J. Am. Chem. Soc. 2004, 126, 14287.

(9) Beebe, J. M.; Engelkes, V. B.; Miller, L. L.; Frisbie, C. D. J. Am. Chem. Soc. 2002, 124, 11268 .

(10) Wierzbinski, E.; Yin, X.; Werling, K.; Waldeck, D. H. J. Phys. Chem. B 2012, $117,4431$.

(11) Wang, G.; Kim, Y.; Choe, M.; Kim, T. W.; Lee, T. Adv. Mat. 2011, 23, 755.

(12) Kim, T. W.; Wang, G. N.; Lee, H.; Lee, T. Nanotechnology 2007, 18.

(13) Milani, F.; Grave, C.; Ferri, V.; Samori, P.; Rampi, M. A. ChemPhysChem 2007, 8,515 .

(14) Weiss, E. A.; Chiechi, R. C.; Kaufman, G. K.; Kriebel, J. K.; Li, Z. F.; Duati, M.; Rampi, M. A.; Whitesides, G. M. J. Am. Chem. Soc. 2007, 129, 4336.

(15) Holmlin, R. E.; Haag, R.; Chabinyc, M. L.; Ismagilov, R. F.; Cohen, A. E.;

Terfort, A.; Rampi, M. A.; Whitesides, G. M. J. Am. Chem. Soc. 2001, 123, 5075.

(16) Simeone, F. C.; Rampi, M. A. CHIMIA 2010, 64, 362.

(17) Nijhuis, C. A.; Reus, W. F.; Barber, J. R.; Dickey, M. D.; Whitesides, G. M. Nano Lett. 2010, 10, 3611.

(18) Reus, W. F.; Nijhuis, C. A.; Barber, J. R.; Thuo, M. M.; Tricard, S.; Whitesides, G. M. J. Phys. Chem. C 2012, 116, 6714.

(19) Thuo, M. M.; Reus, W. F.; Nijhuis, C. A.; Barber, J. R.; Kim, C.; Schulz, M. D.; Whitesides, G. M. J. Am. Chem. Soc. 2011, 133, 2962.

(20) Nijhuis, C. A., Reus, W. F., Barber J. R., Whitesides, G. M. J. Phys. Chem. C 2012, 116, 14139.

(21) Holm, R. Electric Contacts: Theory and Applications; Springer: Berlin, 2001.

(22) Greenwood, J. A., Williamson, J.B.P. Proc. R. Soc. London, Ser. A 1966, 295.

(23) Tabor, D. J. Colloid Interface Sci. 1977, 58.

(24) Slate, P. G. Electrical Contacts: Principles and Applications; Marcel Dekker, Inc.: New York, 1999.

(25) Bowder, F. P.; Tabor, D. The Friction and Lubrication of Solids; Oxford University Press: Oxford, 2001.

(26) Adamson, A. W. Physical Chemistry of Surfaces; John Wiley \& Sons, Inc.: New York, 1997.

(27) Demirel, A. L.; Granick, S. J. Chem. Phys. 1998, 109, 6889. 
(28) Metzger, R. M.; Baldwin, J. W.; Shumate, W. J.; Peterson, I. R.; Mani, P.; Mankey, G. J.; Morris, T.; Szulczewski, G.; Bosi, S.; Prato, M.; Comito, A.; Rubin, Y. J. Phys. Chem. B 2003, 107, 1021.

(29) Stewart, D. R.; Ohlberg, D. A. A.; Beck, P. A.; Chen, Y.; Williams, R. S. Nano Lett. 2004, 4, 133.

(30) Lau, C. N.; Stewart, D. R.; Williams, R. S.; Bockrath, M. Nano Lett. 2004, 4, 569.

(31) Akkerman, H. B.; de Boer, B. J. Phys.: Condens. Matter 2008, 20, 013001.

(32) Salomon, A.; Cahen, D.; Lindsay, S.; Tomfohr, J.; Engelkes, V. B.; Frisbie, C. D. Adv. Mat. 2003, 15, 1881.

(33) Nijhuis, C. A.; Reus, W. F.; Siegel, A. C.; Whitesides, G. M. J. Am. Chem. Soc. 2011, 133, 15397.

17814.

(34) Nijhuis, C. A.; Reus, W. F.; Whitesides, G. M. J. Am. Chem. Soc. 2009, 131, 18386.

(35) Nijhuis, C. A.; Reus, W. F.; Whitesides, G. M. J. Am. Chem. Soc. 2010, 132,

(36) Thuo, M. M.; Reus, W. F.; Simeone, F. C.; Kim, C.; Schulz, M. D.; Yoon, H. J.; Whitesides, G. M. J. Am. Chem. Soc. 2012, 134, 10876.

(37) Yoon, H. J.; Shapiro, N. D.; Park, K. M.; Thuo, M. M.; Soh, S.; Whitesides, G. M. Angew. Chem. Int. Ed. 2012, 51, 4658.

(38) Fracasso, D.; Valkenier, H.; Hummelen, J. C.; Solomon, G. C.; Chiechi, R. C. J. Am. Chem. Soc. 2011, 133, 9556.

(39) Nerngchamnong, N.; Yuan, L.; Qi, D.-C.; Li, J.; Thompson, D.; Nijhuis, C. A. Nature Nanotech. 2013, 8, 113.

(40) Fracasso, D.; Mugladi, M. I.; Rohwerder, M.; Terfort, A.; Chiechi, R. C. J. Phys. Chem. C 2013, IN PRESS.

(41) Cademartiri, L.; Thuo, M. M.; Nijhuis, C. A.; Reus, W. F.; Tricard, S.; Barber, J. R.; Sodhi, R. N. S.; Brodersen, P.; Kim, C.; Chiechi, R. C.; Whitesides, G. M. J. Phys. Chem. C 2012, 116, 10848.

(42) Reus, W. F.; Thuo, M. M.; Shapiro, N. D.; Nijhuis, C. A.; Whitesides, G. M. ACS Nano 2012, 6, 4806.

(43) Dickey, M. D.; Chiechi, R. C.; Larsen, R. J.; Weiss, E. A.; Weitz, D. A.; Whitesides, G. M. Adv. Funct. Mater. 2008, 18, 1097.

(44) Chiechi, R. C.; Weiss, E. A.; Dickey, M. D.; Whitesides, G. M. Angew. Chem. Int. Ed. 2008, 47, 142.

1065.

(45) McCreery, R. L.; Yan, H.; Bergren, A. J. Phys. Chem. Chem. Phys. 2013, 15,

(46) Weiss, E. A.; Kaufman, G. K.; Kriebel, J. K.; Li, Z. F.; Schalek, R.; Whitesides, G. M. Langmuir 2007, 23, 9686.

(47) Akkerman, H. B.; Naber, R. C. G.; Jongbloed, B.; van Hal, P. A.; Blom, P. W. M.; de Leeuw, D. M.; de Boer, B. PNAS 2007, 104, 11161.

(48) Salomon, A.; Boecking, T.; Seitz, O.; Markus, T.; Amy, F.; Chan, C.; Zhao, W.; Cahen, D.; Kahn, A. Adv. Mat. 2007, 19, 445.

(49) York, R. L.; Nguyen, P. T.; Slowinski, K. J. Am. Chem. Soc. 2003, 125, 5948.

(50) Slowinski, K.; Fong, H. K. Y.; Majda, M. J. Am. Chem. Soc. 1999, 121, 7257.

(51) Pourhossein, P.; Chiechi, R. C. ACS Nano 2012, 6, 5566. 
(52) Love, J. C.; Estroff, L. A.; Kriebel, J. K.; Nuzzo, R. G.; Whitesides, G. M. Chem. Rev. 2005, 105, 1103.

(53) Liao, K. C.; Simeone, F. C.; Whitesides, G. M. unpublished observations.

(54) Huisman, E. H.; Guedon, C. M.; van Wees, B. J.; van der Molen, S. J. Nano Lett. 2009, 9, 3909. 


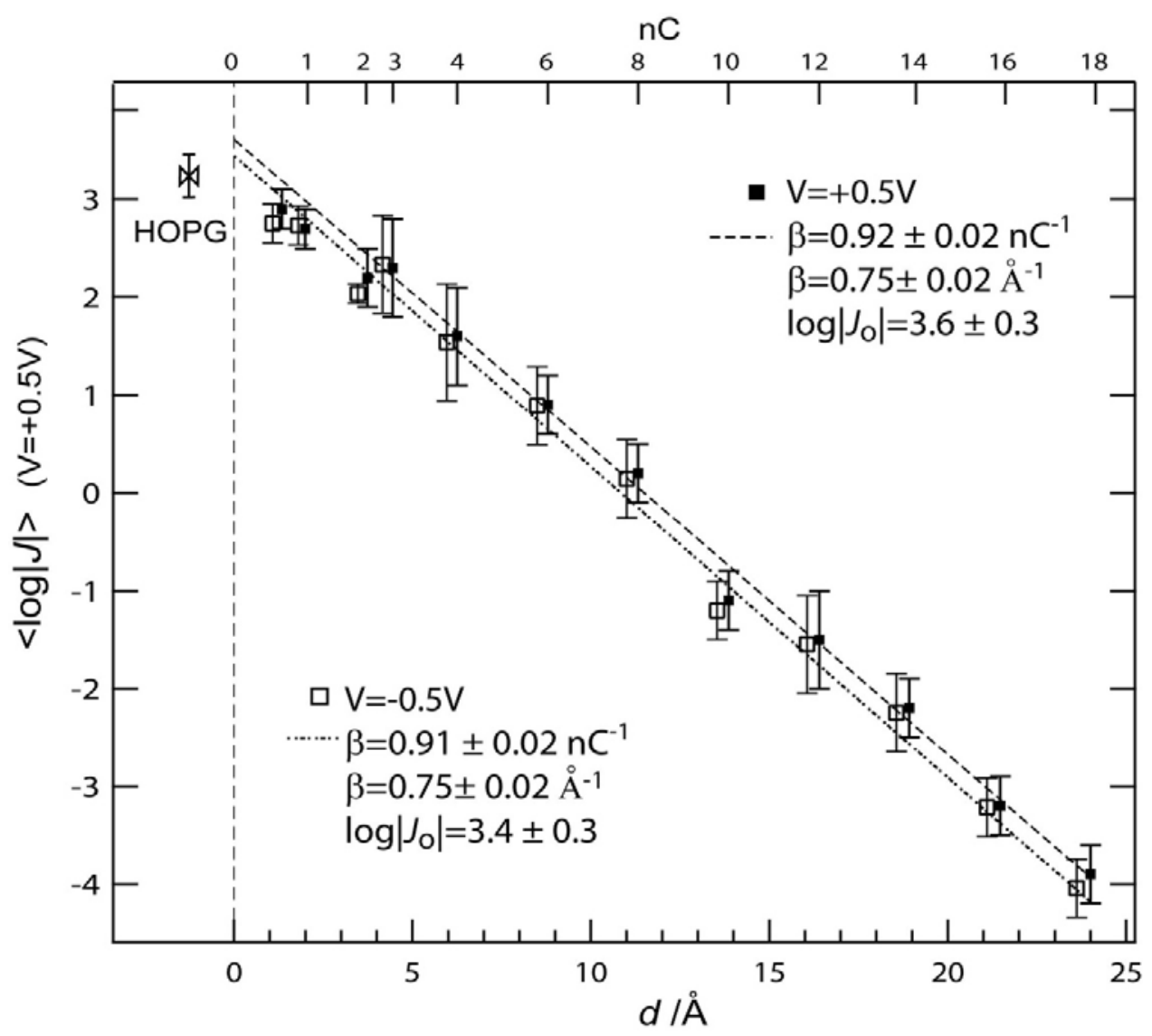

Figure 1. Plot of the Gaussian mean values $<\log |J|>$ through SAMs of $n$-alkanethiolates on $\mathrm{Ag}^{\mathrm{TS}}$ substrates versus the length $d$ of the tunnel gap established by the alkyl chain. Assuming a through-molecule tunneling, $d$ was measured as the distance in $\AA$ from the S-terminus of the $n$ alkanethiolates (in their all trans configuration) to the distal $\mathrm{H}$ atom closest to the top electrode. On the top-axis, $d$ is given as number $(\mathrm{nC})$ of $\mathrm{C}$ atoms of the molecular backbone. Dashed lines represents the linear regression analyses, which give $\beta / 2.303$ (slopes) and $\log \left|J_{o}\right|$ (intercepts at $d=0$ ). "HOPG" indicates the logarithm of the conductance of $\mathrm{Ga}_{2} \mathrm{O}_{3}$ estimated from $\mathrm{HOPG} / / \mathrm{Ga}_{2} \mathrm{O}_{3} / \mathrm{EGaIn}$ junction. 


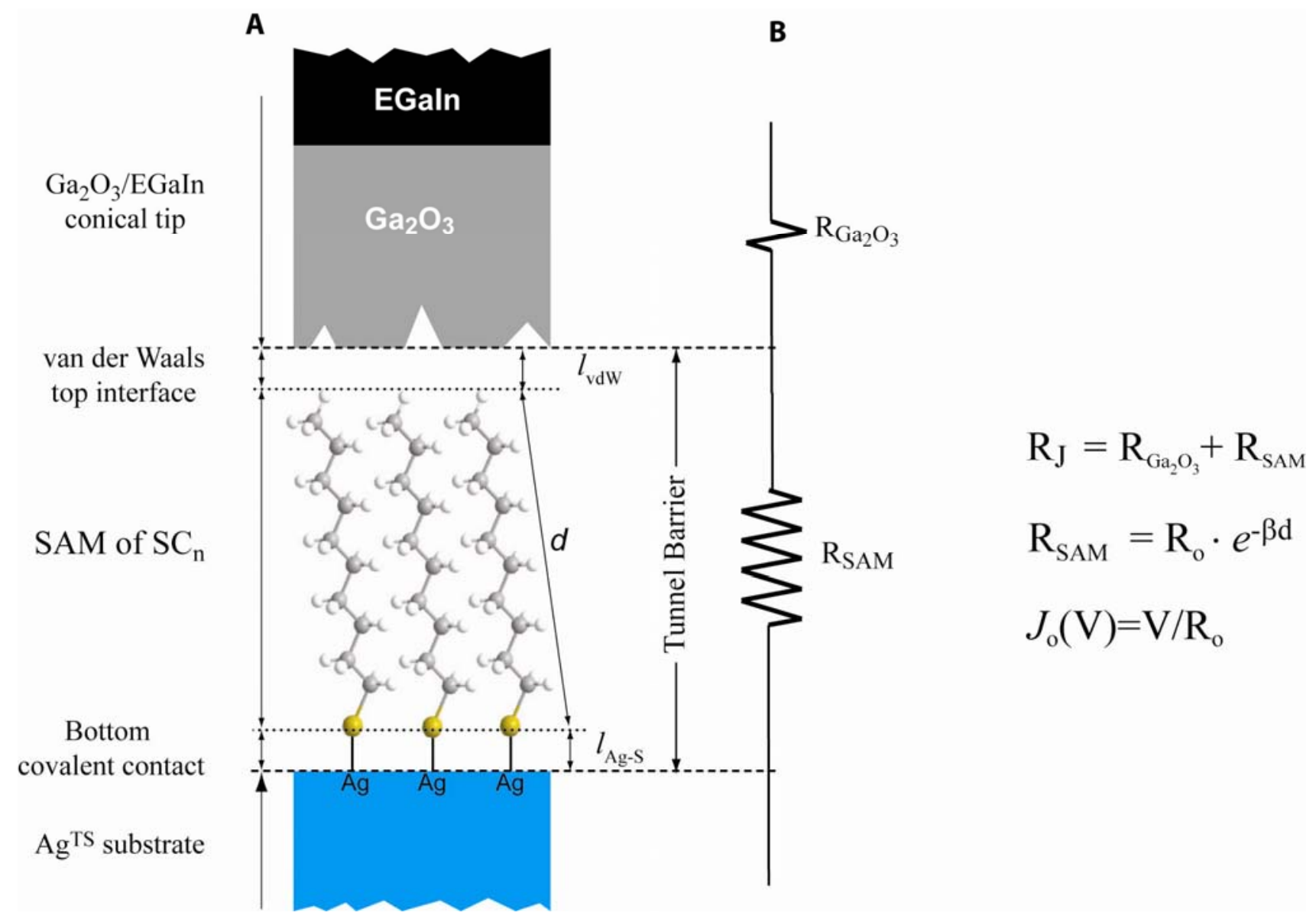

Figure 2. A) Structure of $\mathrm{Ag}^{\mathrm{TS}}-\mathrm{SAM} / / \mathrm{Ga}_{2} \mathrm{O}_{3} / \mathrm{EGaIn}$ junctions. B) Components of the resistance of the junction $\left(\mathrm{R}_{\mathrm{j}}\right): R_{\mathrm{SAM}}$, tunneling resistance across the SAM; $R_{\mathrm{Ga}_{2} \mathrm{O}_{3}}$, resistance of the $\mathrm{Ga}_{2} \mathrm{O}_{3}$ layer. $l_{A g-S}$ is the length of the bottom $\mathrm{Ag}^{\mathrm{TS}}$-S covalent contact; $l_{v d W}$ is the thickness of the top $\mathrm{SAM} / / \mathrm{Ga}_{2} \mathrm{O}_{3}$ van der Waals interface; $d$ is the length of the tunnel barrier established by the alkyl chain. Assuming a through-bond mechanism, $d$ coincides with the length of the alkyl chain. $J_{o}$ is defined as the current across a junction with $d=0$. 

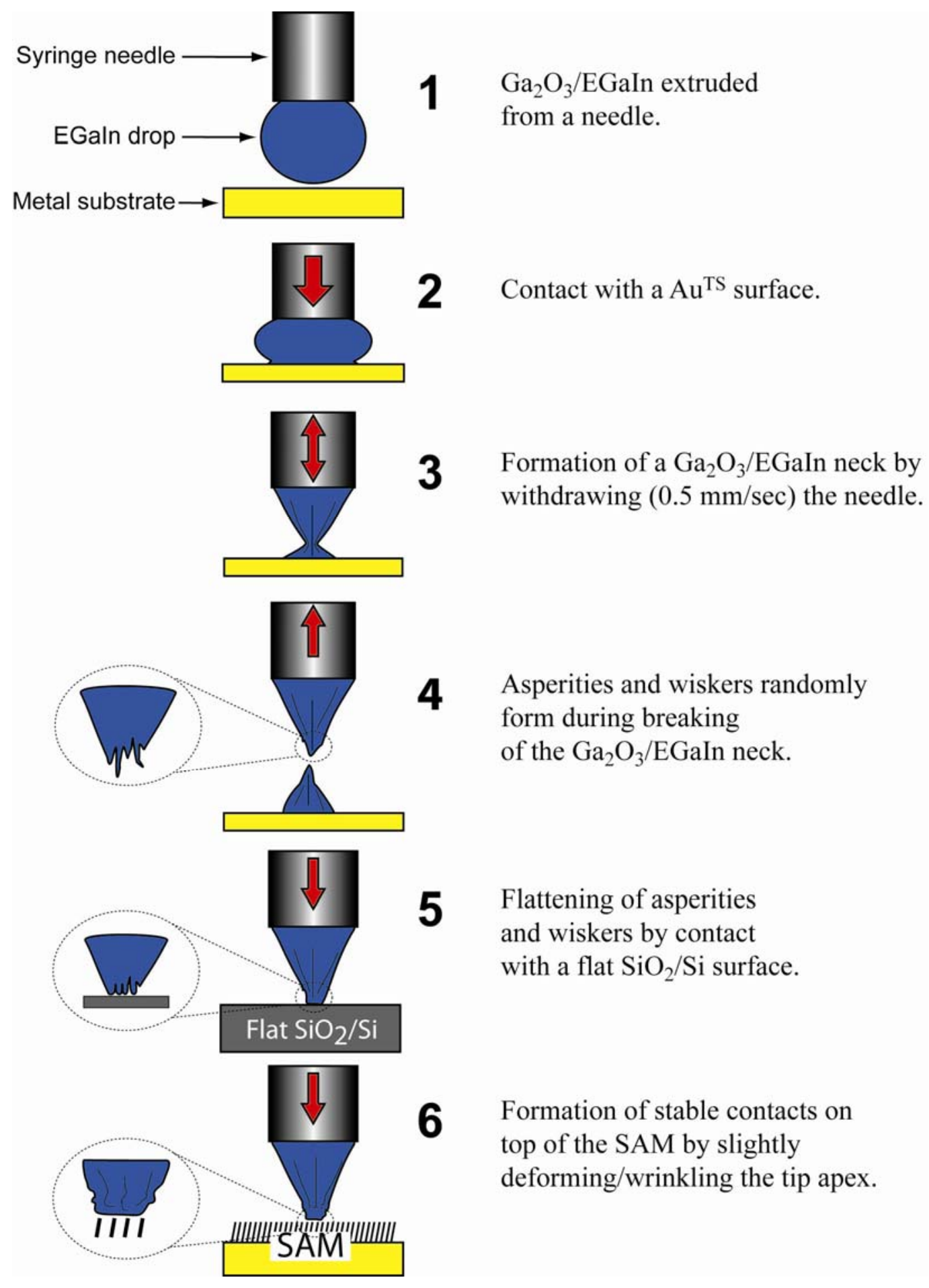

Figure 3. Procedure for the fabrication of pre-flattened $\mathrm{Ga}_{2} \mathrm{O}_{3} / \mathrm{EGaIn}$ conical tips. Flattening is achieved by contact of the tip with a flat $\mathrm{Si} / \mathrm{SiO}_{2}$ surface (step 5). In Step 4, the sizes of asperities were exaggerated for clarity. A video of this procedure is available as supporting material. 

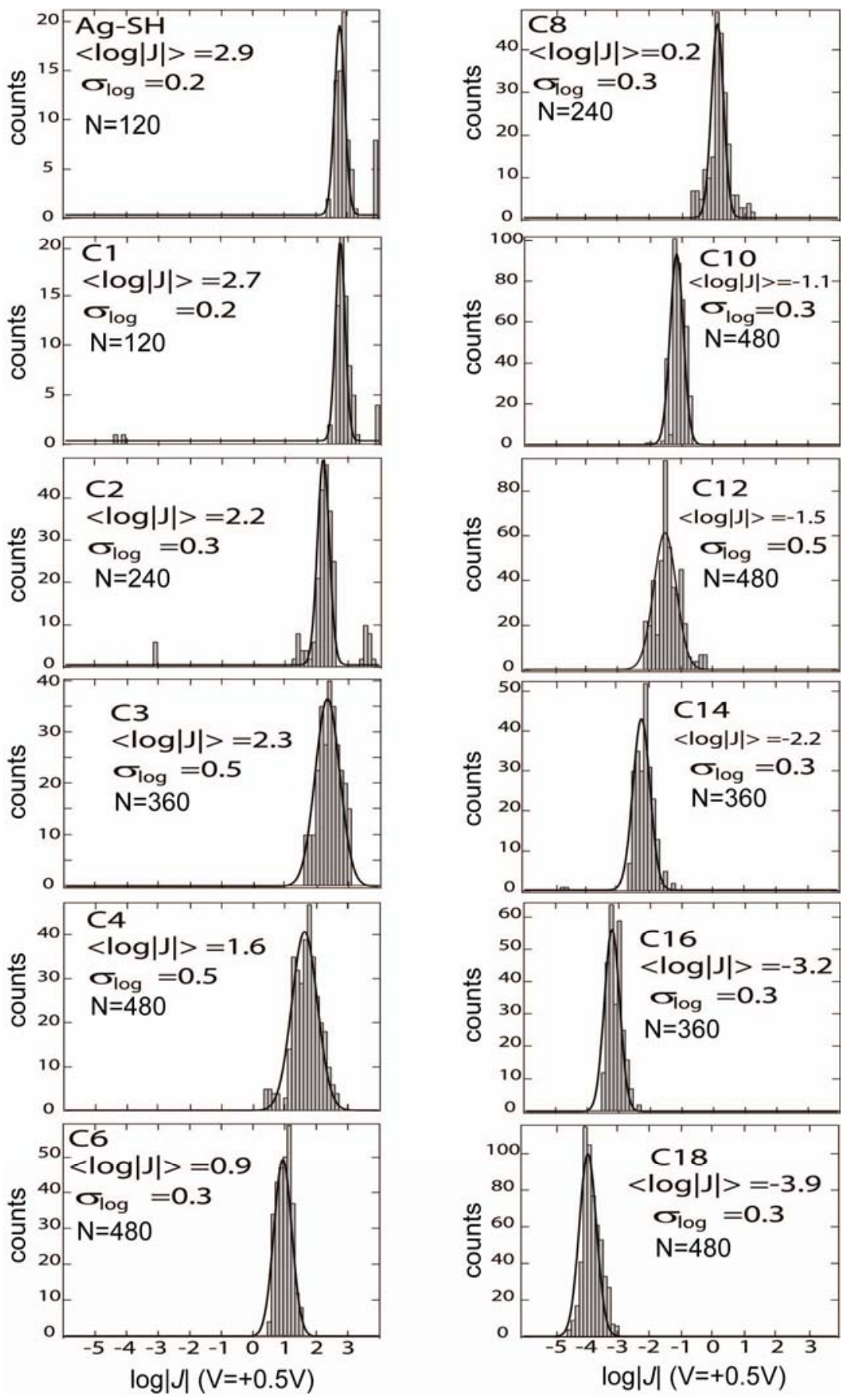

Figure 4. Histograms showing the distributions of values of $\log |J|$ at $\mathrm{V}=+0.5 \mathrm{~V}$ across $\mathrm{Ag}^{\mathrm{TS}}$ $\mathrm{SAM} / / \mathrm{Ga}_{2} \mathrm{O}_{3} / \mathrm{EGaIn}$ junctions using flattened conical tips. With "Cn" we indicate SAMs of alkanethiolates with $\mathrm{n}$ carbon atoms. Solid curves represent Gaussian fits. $\mathrm{N}$ is the number of data points. Data collected using the $1 / 3 / 20$ protocol. No data were excluded. The heights of the bars of the histograms count the number of points (counts) with values of $\log |J|$ within the width (0.13) of the bars. For each distribution, the value of Gaussian mean, median, and mode (the value of $\log |J|$ with the highest bar) differed by less than 0.1 . 


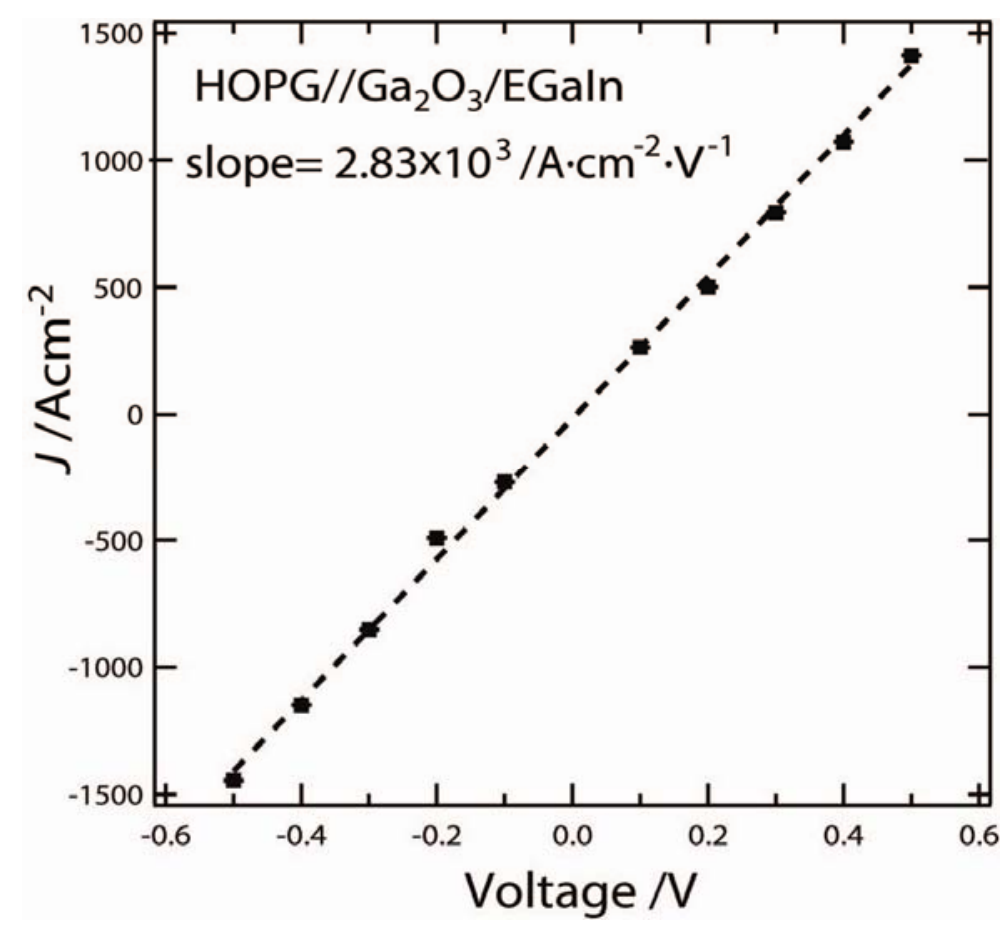

Figure 5. Average $J-\mathrm{V}$ curve for $\mathrm{HOPG} / / \mathrm{Ga}_{2} \mathrm{O}_{3} / \mathrm{EGaIn}$ junction. The slope estimates the specific conductivity (conductivity per unit area) of the $\mathrm{Ga}_{2} \mathrm{O}_{3}$ layer of $\mathrm{Ga}_{2} \mathrm{O}_{3} / \mathrm{EGaIn}$ conical tips. 

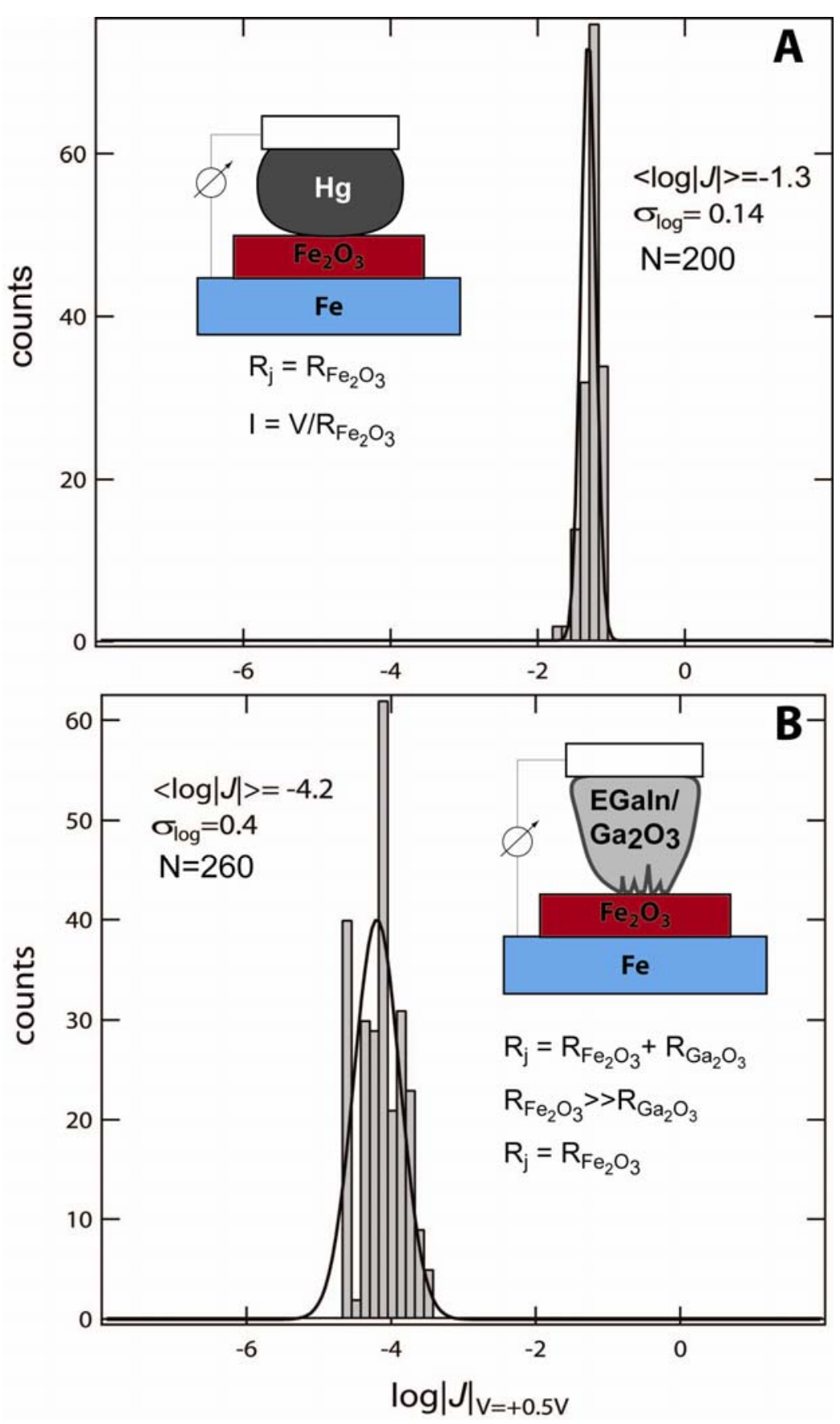

Figure 6. Histograms of the values of $\log |J|$ at $\mathrm{V}=+0.5 \mathrm{~V}$ across: A) $\mathrm{Fe} / \mathrm{Fe}_{2} \mathrm{O}_{3} / / \mathrm{Hg}$ junctions; and B) $\mathrm{Fe} / \mathrm{Fe}_{2} \mathrm{O}_{3} / / \mathrm{Ga}_{2} \mathrm{O}_{3} / \mathrm{EGaIn}$ junctions. Solid lines represent Gaussian best fits. We used the same $\mathrm{Fe} / \mathrm{Fe}_{2} \mathrm{O}_{3}$ substrate for all measurements. The resistances $\left(\mathrm{R}_{\mathrm{J}}\right)$ of junctions $\mathrm{A}$ and $\mathrm{B}$ were approximately the same $\left(\mathrm{R}_{\mathrm{Ga} 2 \mathrm{O} 3}\right)$ by experimental design. 

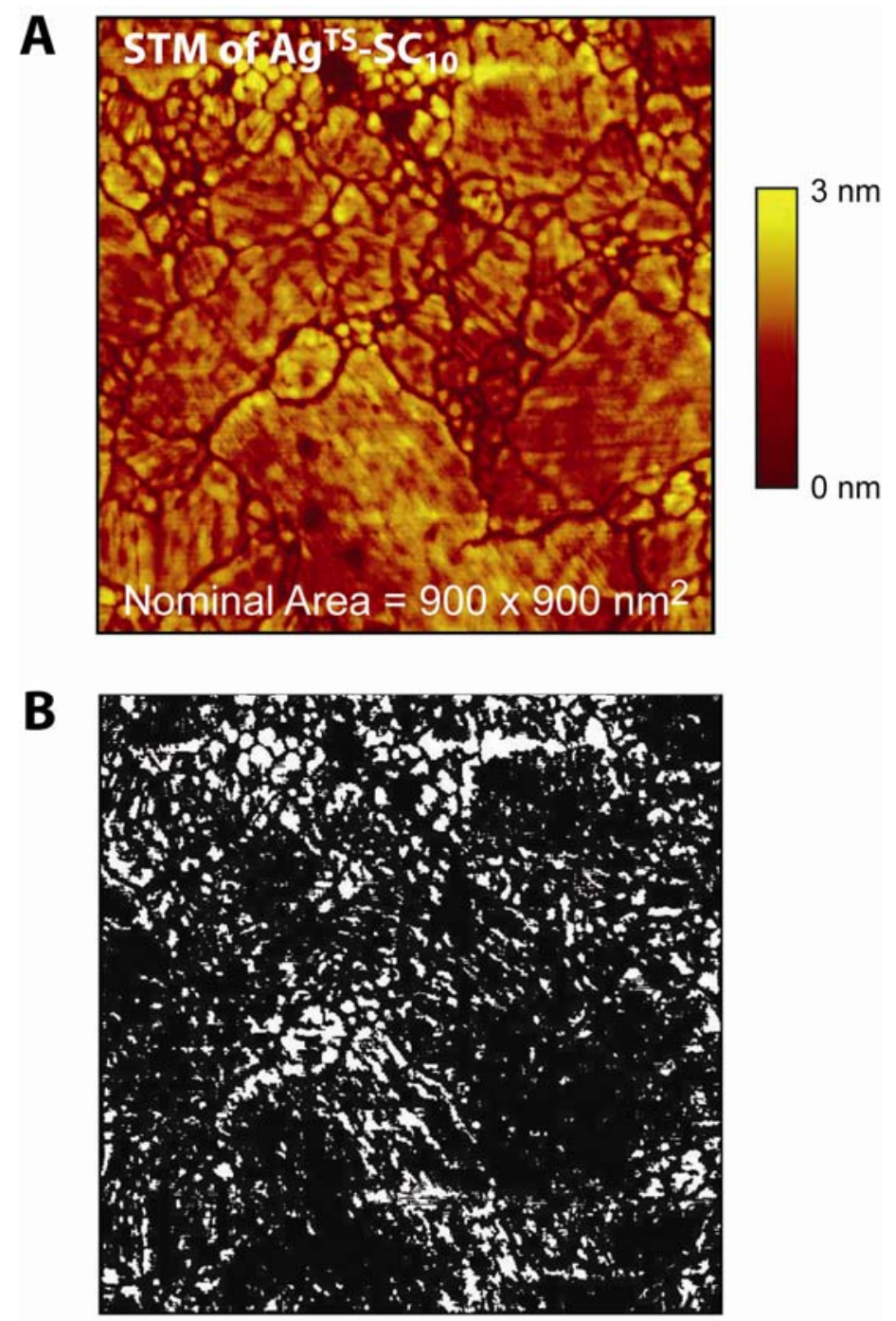

Fraction for Contact, $\alpha_{\text {SAM }}=12 \%$

Figure 7. A) Scanning Tunneling Microscopy image of a $\mathrm{Ag}^{\mathrm{TS}}-\mathrm{SC}_{10}$ substrate. B) Surface area available for contact (white area) estimated via digital analysis of the STM image in A. The contact area was estimated as the number of pixel within $2 \AA$ from the top-most average plane of the image. (Lateral profiles of the STM images are reported in Figure S3) 


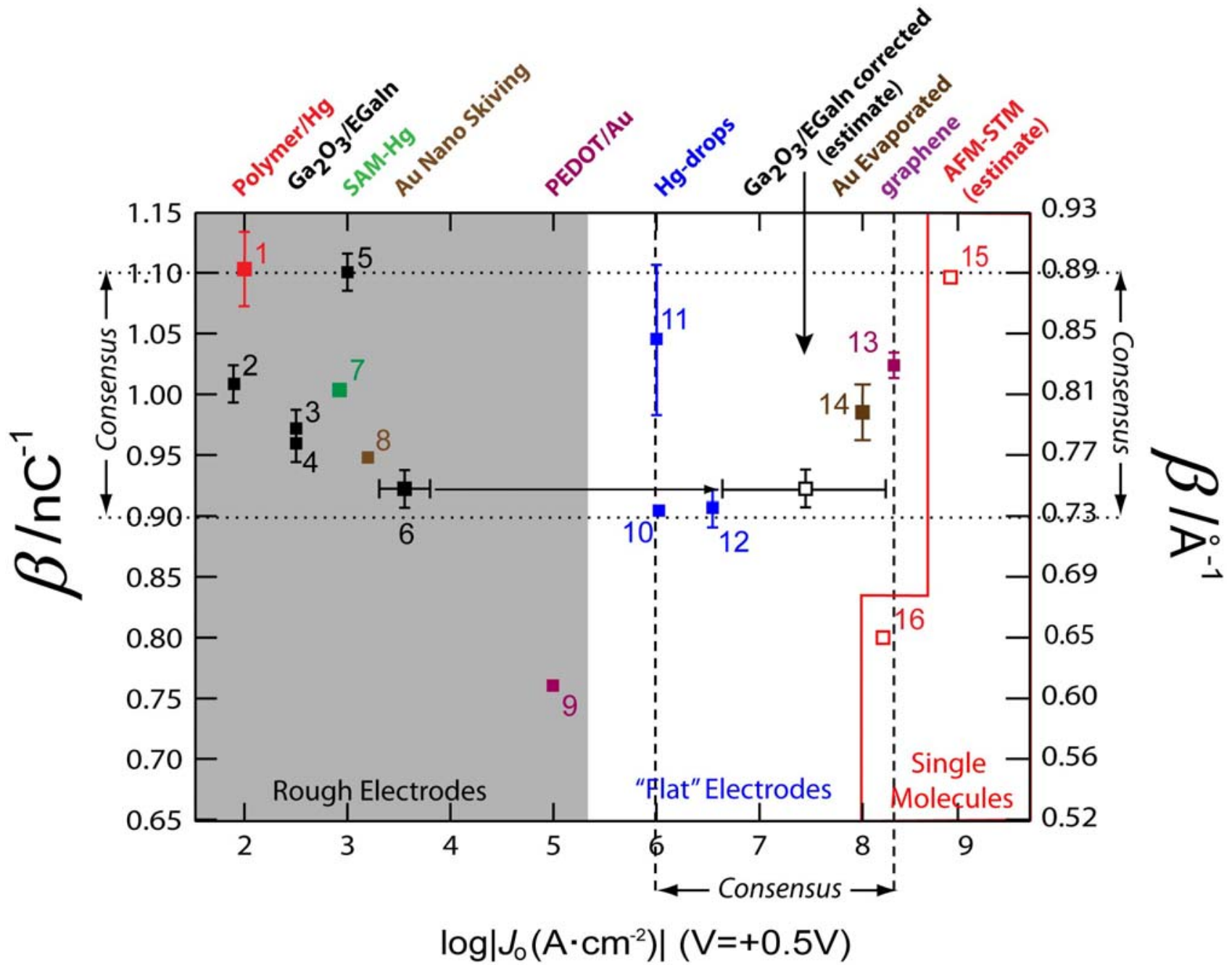

Figure 8. Plot of values of $\beta$ versus values of $\log \left|J_{o}(\mathrm{~V}=+0.5 \mathrm{~V})\right|$ reported (filled dots) or estimated (empty squares) for tunneling through $n$-alkanethiolates with junctions using topelectrodes indicated on the top-axis. Numbering from Table 1. The horizontal arrowed line points to the value of $J_{o}$ for $\mathrm{Ag}^{\mathrm{TS}}-\mathrm{SAM} / / \mathrm{Ga}_{2} \mathrm{O}_{3} / \mathrm{EGaIn}$ corrected for the value of the effective electrical contact area $\left(\mathrm{A}_{\text {elec }}=10^{-4 \pm 0.5} \cdot \mathrm{A}_{\mathrm{geo}}\right)$; the horizontal error bar of this estimate incorporates the uncertainties on the estimation of $J_{o}$ from tunneling across SAMs, and on the estimation of the effective electrical contact area. Junctions grouped in the gray zone use either rough topelectrodes, or rough bottom-electrodes. 